\title{
Local perturbations of periodic systems. Chemisorption and point defects by GoGreEnGo.
}

\author{
Ilya V. Popov ${ }^{1}$, Timofei S. Kushnir ${ }^{1}$, Andrei L. Tchougréeff ${ }^{1}$
}

June 20, 2021

1 - A.N. Frumkin Institute of Physical Chemistry and Electrochemistry RAS, Moscow, Russia

\begin{abstract}
We present a software package GoGREEnGo - an overlay aimed to model local perturbations of periodic systems due to either chemisorption or point defects. The electronic structure of an ideal crystal is obtained by worldwide distributed standard quantum physics/chemistry codes, then processed by various tools performing projection to atomic orbital basis sets. Starting from this, the perturbation is addressed by GoGreenGo with use of the Green's functions formalism, which allows to evaluate its effect on the electronic structure, density matrix and energy of the system. In the present contribution the main accent is made on processes of chemical nature such as chemisorption or doping. We address a general theory and its computational implementation supported by a series of test calculations for benchmark model solids: simple, face-centered and body-centered cubium systems. In addition, more realistic problems of local perturbations in graphene lattice such as lattice substitution, vacancy and "on-top" chemisorption are considered.
\end{abstract}

Keywords: Point defects in crystals, electronic structure, chemisorption, substitutional defects, Green's functions 


\section{IN GREEN WE TRUST}

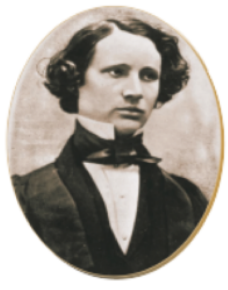

Point defects in crystals form a wide class of processes being of great importance in solid state chemistry. Only by considering surface chemistry one can propose a numerous examples - from formation of isolated surface defects to a single particle chemisorption and elementary reactions on catalysts' surfaces. Theoretical investigation of these processes, aiming to understand their mechanisms from the electronic structure perspective, presents one of many important branches of solid state chemistry deserving close attention. In this work we present a new software package GoGREENGo specifically designed to perform computationally effective quantum chemical calculations of local processes in solids and to provide results in "chemical" terms. 


\section{Introduction}

A great number of elementary processes of significant importance in solid state chemistry are local in nature [1], although they take place in a "matrix" of crystal (periodic) system. Currently, local processes/effects in solids are addressed by using standard computational chemistry codes pushing towards the limits of their applicability with a considerable loss of efficiency. Indeed, applying "molecular" codes to study a point feature in a crystal requires tricky setting up of boundary conditions in the cluster models of eventually infinite systems [2]. Any way, one has to consider rather large clusters to compensate boundary effects. At the same time, the required embedding procedures are sometimes awkward and not unique, particularly in the case of clusters cut from ionic crystals or metals. A comprehensive review of the related problems is given in [3].

An alternative and nowadays predominant approach is to accommodate periodic wave function [4] or PAW-DFT [5] based methods to study local effects. In order to do so, one needs to increase the size of the unit cell to ensure correct proportion of the point defects and to minimize lateral interactions between them (see, for example, Refs. [6,7] and references therein). The unit cell enlargement increases the computational costs drastically with the scaling up to $O\left(N^{3}\right)$, where $N$ is the "size" of the unit cell. The entire study consists of full calculations of the original (ideal) periodic system and repeating them for as well periodic models which include intrinsically local features. The effect of the local perturbations is then derived by comparing results of these calculations.

Apparently, the state of the locally perturbed crystal cannot differ too much from that of the ideal one. The mentioned standard approaches do not take this fact into account, although it seems to be profitable to avoid repetitive recalculations from the scratch and to make a better use of the eventually more precise information about the electronic structure of the ideal crystal. Based on this idea, further embedding schemes had been developed (for example Refs. $[8,9,10]$ ) which employ the information about the ideal crystal for analysis of the defect ones. Also, these schemes are not free from numerous ad hoc assumptions leading to an uncontrollable loss of precision and predictive capacity.

Remarkably, a detailed theory of local perturbations of periodic systems using the Green's function (GF) formalism had been developed yet in 60-ies [11, 12, 13, 14] (based on an earlier work [15]) and was applied to chemisorption in numerous works $[16,17,18,19,20,21,22,23,24,25,26,27]$. The most characteristic feature of this approach is that starting from a solution of the ideal periodic problem and from its local perturbation, one obtains the answer as a correction to the unperturbed solution. By this, (i) the highly inefficient step of solving the perturbed problem from the scratch is avoided and (ii) the result is a pure effect of the perturbation. The theory is highly pedagogically explained in Ref. [28], where it is used to provide pictorial description of the electronic structure perturbations occurring throughout chemisorption. Otherwise more mathematical, but still suitable for a chemistry theory student exposition of the required apparatus is given in Ref. [29].

Being actively used in 70-ies to study local defects in rather simple model solids, the GF theory was practically abandoned later and replaced by supercell periodic and finite clusters calculations [30]. Undeservedly forgotten, this approach has been getting much less attention since then, especially in the community of solid state chemistry. ${ }^{1}$ Although, it suits better for discussing chemical problems, it has never enjoyed any generalization to more realistic systems described by rigorously defined Hamiltonians. As well, there is no reported software implementing the general theory, which would be suitable for a broad range of solid state and surface chemistry problems (with different kinds of defects in crystals and/or chemisorption). The purpose of the present work and of the proposed GoGREENGo software package is to incorporate the mentioned approach into a context of modern theoretical methodology used in solid state chemistry. Since we mainly focus on the chemical interpretation of electronic structure calculations (described in "chemical" terms such as atomic charges or bond orders), it is implemented for basis of local atomic orbitals.

The paper is organized as follows. In the Theory and Implementation Sections we sketch the generalized theory and implementation details of the programs included in the GoGREEnGo package. After that we provide test results for various benchmarks by considering substitutional and interstitial defects in the model cubic lattices. Finally, GoGreEnGo package is applied to tackle more realistic problems devoted to

\footnotetext{
${ }^{1}$ Relatively modern application of GFs to the problem of point defects in crystals was developed in the framework of KorringaKohn-Rostoker method (KKR-GF) [31], which is based on ideas of multiple-scattering theory and, consequently, relies on rather different formulation of the GF theory compared to the one used in above cited references and in the present paper (see below).
} 
graphene. One can find more detailed specifications of the GoGreEnGo by following the link [32]. The Supporting Material collects analytical results used for the testing.

\section{Theory Sketch}

\section{Structure of the electronic problem}

Theoretical basis of the proposed software development is the self-consistent perturbation theory of manyelectron systems [33]. It starts from representing the electronic structure either by a single Slater determinant formed by one-electron (spin-)orbitals for the wave function based methods (as exemplified by e.g. [4, 34, $35]$ ) or by one-electron density in the DFT context. In the latter case one-electron orbitals reappear as Kohn-Sham ones, so that either wave-function or (Kohn-Sham) DFT procedures can be represented as an iterative eigenvalue-eigenvector problem with some generalized density dependent Fockian matrix $\mathbf{F}[\mathbf{P}]$ in the functional space spanned either by exclusively atomic states or by an assembly of plane waves and atomic local states augmenting (PAW) the former:

$$
(\varepsilon \mathbf{I}-\mathbf{F}[\mathbf{P}])|\lambda\rangle=0 .
$$

Solutions of eq. (1) are the eigenvalue-eigenvector pairs $\varepsilon_{\lambda},|\lambda\rangle$ numbered by assemblies of quantum numbers $\lambda$ and satisfying the well known relations:

$$
\mathbf{F}[\mathbf{P}]|\lambda\rangle=\varepsilon_{\lambda}|\lambda\rangle .
$$

The density $\mathbf{P}$ is determined by the occupied eigenvectors $|\lambda\rangle$ whose eigenvalues are subject to the condition $\varepsilon_{\lambda} \leq \varepsilon_{F}$, where $\varepsilon_{F}$ is the Fermi energy selected so that the number of occupied one-electron states equals to that of electrons (Fermi statistics). The eigenvalue-eigenvector problems eqs. (1), (2) are sequentially solved until the convergence for $\mathbf{P}$ is achieved.

\section{Green's function's representation}

The eigenvalue-eigenvector problem can be alternatively formulated in terms of the quantity ${ }^{2}$ :

$$
\mathbf{G}(z)=(z \mathbf{I}-\mathbf{F})^{-1},
$$

the famous Green's function of a complex argument $z$. Since the Fockian $\mathbf{F}$ (hereinafter, we omit its $\mathbf{P}$ dependence for brevity) is a Hermitian operator, its eigenvalues $\varepsilon_{\lambda}$ are always real. Thus, for an arbitrary complex $z$ unequal to any of $\varepsilon_{\lambda}$ the matrix $(z \mathbf{I}-\mathbf{F})$ is nondegenerate and can be inverted producing a $z$ dependent quantity eq. (3). Its closest relation to the eigenvalue-eigenvector problem stems from the spectral representation:

$$
\mathbf{G}(z)=\sum_{\lambda} \frac{|\lambda\rangle\langle\lambda|}{z-\varepsilon_{\lambda}}
$$

which immediately derives from the expressions for the identity matrix and the Fockian in the basis of its eigenvectors:

$$
\mathbf{I}=\sum_{\lambda}|\lambda\rangle\left\langle\lambda\left|; \mathbf{F}=\sum_{\lambda} \varepsilon_{\lambda}\right| \lambda\right\rangle\langle\lambda|
$$

As soon as the GF is known in the basis of the eigenvalues of $\mathbf{F}$, where it is diagonal, it is known in whatever basis. E.g. in the basis of local atomic spin-orbitals $|a\rangle,|b\rangle, \ldots$ a Greenian matrix is formed by the elements

$$
G_{a b}(z)=\sum_{\lambda} \frac{\langle a \mid \lambda\rangle\langle\lambda \mid b\rangle}{z-\varepsilon_{\lambda}}
$$

where $\langle a \mid \lambda\rangle$ are expansion coefficients of the eigenvector $|\lambda\rangle$ over atomic basis. ${ }^{3}$

\footnotetext{
${ }^{2}$ Numerous sources are available, in the computational chemistry context Refs. [28, 29] can be recommended.

${ }^{3}$ The bra-ket notation is used (see, e.g. Ref. [36]).
} 
Being defined as a function of complex variable, GF appears in the expressions for the physical quantities under the integral over the real axis only. Since GF has a lot of poles on the real axis, it should be considered there as a distribution (or a generalized function). Such distribution is defined as a limit :

$$
\mathbf{G}(\varepsilon)=\lim _{\nu \rightarrow 0^{+}} \mathbf{G}(\varepsilon+i \nu),
$$

where $z$ is set to be $\varepsilon+i \nu$ with real $\varepsilon$ and $\nu$. As described in Appendix A, evaluation of the limit entering the later equation leads to the following GF on the real axis

$$
\begin{aligned}
\mathbf{G}(\varepsilon) & =\Re \mathbf{G}(\varepsilon)+i \Im \mathbf{G}(\varepsilon), \\
\Re \mathbf{G}(\varepsilon) & =\sum_{\lambda}|\lambda\rangle\langle\lambda| \mathscr{P}\left(\frac{1}{\varepsilon-\varepsilon_{\lambda}}\right), \\
\Im \mathbf{G}(\varepsilon) & =-\pi \sum_{\lambda}|\lambda\rangle\langle\lambda| \delta\left(\varepsilon-\varepsilon_{\lambda}\right),
\end{aligned}
$$

where $\delta(\varepsilon)$ is the Dirac $\delta$-"function" and $\mathscr{P}$ indicates that the integral of a function $f(\varepsilon)$, multiplied by $\left(\varepsilon-\varepsilon_{\lambda}\right)^{-1}$, with respect to $\varepsilon$ must be understood as the Cauchy principal value. In terms of the GF of a real argument the general expression for the density operator (eq. (29) of Appendix A) takes the form:

$$
\mathbf{P}=-\pi^{-1} \int_{-\infty}^{\varepsilon_{F}} \Im \mathbf{G}(\varepsilon) d \varepsilon .
$$

\section{Perturbations in terms of Green's function}

The GF formalism as sketched in the previous Subsection does not add too much to the usual treatment of the eigenvalue-eigenvector problem. Its power manifests itself when the perturbations are addressed. For the GF of the Fockian $\mathbf{F}$, being a sum of an unperturbed one $\mathbf{F}^{(0)}$ and a perturbation $\mathbf{F}^{\prime}$ :

$$
\mathbf{F}=\mathbf{F}^{(0)}+\mathbf{F}^{\prime},
$$

the Dyson equation

$$
\mathbf{G}(z)=\mathbf{G}^{(0)}(z)+\mathbf{G}^{(0)}(z) \mathbf{F}^{\prime} \mathbf{G}(z)
$$

holds [37]. Being solved for $\mathbf{G}(z)$, it gives:

$$
\mathbf{G}(z)=\left(\mathbf{I}-\mathbf{G}^{(0)}(z) \mathbf{F}^{\prime}\right)^{-1} \mathbf{G}^{(0)}(z),
$$

which generates the perturbation series if one expands the inverse matrix in the geometric series:

$$
\begin{aligned}
\left(\mathbf{I}-\mathbf{G}^{(0)}(z) \mathbf{F}^{\prime}\right)^{-1} & =\mathbf{I}+\mathbf{G}^{(0)}(z) \mathbf{F}^{\prime}+\mathbf{G}^{(0)}(z) \mathbf{F}^{\prime} \mathbf{G}^{(0)}(z) \mathbf{F}^{\prime}+\ldots \\
\mathbf{G}(z) & =\mathbf{G}^{(0)}(z)+\mathbf{G}^{(0)}(z) \mathbf{F}^{\prime} \mathbf{G}^{(0)}(z)+\mathbf{G}^{(0)}(z) \mathbf{F}^{\prime} \mathbf{G}^{(0)}(z) \mathbf{F}^{\prime} \mathbf{G}^{(0)}(z)+\ldots
\end{aligned}
$$

Thus, the general perturbative treatment rewrites in terms of the Green's functions. Formally, in the case of a point defect in an infinite crystal the solution of the Dyson equation would require inversion of a matrix of infinite dimension. However, switching to a local atomic orbital representation allows to reduce the problem to a finite one, since in this case a point (local) perturbation acts on a relatively low-dimensional subspace $(P)$ of the entire space of one-electronic states. In GoGreenGo we employ this possibility and consider the perturbation matrices of a form:

$$
\mathbf{F}^{\prime}=\left(\begin{array}{cc}
\mathbf{F}_{P P}^{\prime} & 0 \\
0 & 0
\end{array}\right)=\left(\begin{array}{ll}
\mathbf{V} & 0 \\
0 & 0
\end{array}\right) .
$$

The Greenian matrix is then split in blocks: 


$$
\mathbf{G}^{(0)}=\left(\begin{array}{ll}
\mathbf{G}_{P P}^{(0)} & \mathbf{G}_{P Q}^{(0)} \\
\mathbf{G}_{Q P}^{(0)} & \mathbf{G}_{Q Q}^{(0)}
\end{array}\right),
$$

where $Q$ refers to the orthogonal complement of the subspace $P$ (argument $z$ is omitted for brevity). Introducing a $\operatorname{dim} P \times \operatorname{dim} P$ matrix $\mathbf{M}:^{4}$

$$
\mathbf{M}=\mathbf{V}\left(\mathbf{I}_{P P}-\mathbf{G}_{P P}^{(0)} \mathbf{V}\right)^{-1}
$$

and following the procedure given in Appendix B, one obtains the corrections to the matrix blocks:

$$
\begin{gathered}
\mathbf{G}_{P P}-\mathbf{G}_{P P}^{(0)}=\mathbf{G}_{P P}^{(0)} \mathbf{M G}_{P P}^{(0)}, \\
\mathbf{G}_{Q Q}-\mathbf{G}_{Q Q}^{(0)}=\mathbf{G}_{Q P}^{(0)} \mathbf{M G}_{P Q}^{(0)}, \\
\mathbf{G}_{P Q}-\mathbf{G}_{P Q}^{(0)}=\mathbf{G}_{P P}^{(0)} \mathbf{M} \mathbf{G}_{P Q}^{(0)} \quad, \quad \mathbf{G}_{Q P}-\mathbf{G}_{Q P}^{(0)}=\mathbf{G}_{Q P}^{(0)} \mathbf{M} \mathbf{G}_{P P}^{(0)},
\end{gathered}
$$

which, respectively, express the effect of the perturbation on the Green's function in the subspace $P$ itself, in the subspace where the perturbation is absent $(Q)$ and on the coupling between the perturbed and unperturbed subspaces. Density matrix elements of the perturbed system are calculated with eq. (8) once the Dyson equation is solved. If one takes into account the density dependence of the Fockian, the calculated density serves as an input for a next step of iterative solution. This setting is referred below as the selfconsistent one.

\section{Specific of the problems at hand}

So far reviewed greenistic representation of the eigenvalue-eigenvector problem is fairly general. Here, we apply it to the crystal point defects of different kinds. We consider two types of such defects: (i) point-wise perturbation of the crystalline matrix itself - the lattice substitution and the vacancy; and (ii) interaction of the crystal with an extra "particle" - dubbed in different contexts as an interstitial defect or an adsorbate. In either case we shall employ the generic term defect. The unperturbed solution described by GF $\mathbf{G}^{(0)}$ of the ideal crystal (and of the finite adsorbate in the case (ii)) is assumed to be known. Below we briefly review the specific of the greeninstic formalism as applied to infinite periodic systems with point defects.

\section{Green's functions of crystals}

When it comes to solids, the solution of the eigenvalue-eigenvector problem has specifics described in handbooks on solid state physics and chemistry [38] (see chapters devoted to tight binding approximation). Due to translation invariance of an ideal infinite crystal, its Fockian accepts the block-diagonal form in the basis of the Bloch sums:

$$
|a k\rangle=\frac{1}{\sqrt{\mathrm{K}}} \sum_{r} \exp (i k r)|a r\rangle
$$

of $A$ atomic states. Here $A$ is the number of atomic spin-orbitals $a=1 \div A, r$ stands for a unit cell index and $\mathrm{K}$ is the number of $k$-points involved in the calculation. ${ }^{5}$ The blocks are numbered by the wave vectors $k$ from the first Brillouin zone and respective eigenvalues form $A$ functions $\varepsilon_{\alpha k}$ of $k$ ( $\left.\alpha=1 \div A\right)$ - (electronic) bands with the eigenvector expansion coefficients forming $k$-dependent $A \times A$ matrices with elements $\langle\alpha k \mid a k\rangle$. In this context, the generalized quantum number $\lambda$, labeling the eigenvalues of the Fockian, splits in the pair of the wave vector $k$ and the band index $\alpha: \lambda=(\alpha, k)$. Thus, the GF of the ideal crystal with $A$ bands reads:

$$
\mathbf{G}(z)=\sum_{\alpha k} \frac{|\alpha k\rangle\langle\alpha k|}{z-\varepsilon_{\alpha k}} .
$$

It is as well block-diagonal with $A \times A$ blocks numbered by $k$. A remarkable feature of the "theoretical" GF of the crystal is that the poles coalesce in (quasi-) continuous segments being the allowed energy bands of

\footnotetext{
${ }^{4}$ sometimes called a mass operator.

${ }^{5}$ It is equal to the number of unit cells used in a periodic model of the infinite crystal [39].
} 
electrons (see [38]). Considering the GF on the real axis according to eq. (7) produces the electronic density of states (DOS):

$$
\operatorname{DOS}(\varepsilon)=-\frac{1}{\pi \mathrm{K}} \operatorname{Sp} \Im \mathbf{G}(\varepsilon)=\sum_{\alpha k} \delta\left(\varepsilon-\varepsilon_{\alpha k}\right),
$$

familiar to the workers of the field.

Applying the same trick to the diagonal elements of the Greenian matrix eq. (5) in the basis of local atomic orbitals

$$
G_{a a}(z)=\sum_{\alpha k} \frac{\langle a k \mid \alpha k\rangle\langle\alpha k \mid a k\rangle}{z-\varepsilon_{\alpha k}}
$$

we arrive to

$$
\operatorname{DOS}_{a}(\varepsilon)=-\frac{1}{\pi \mathrm{K}} \Im G_{a a}(\varepsilon)=\sum_{\alpha k}\langle a k \mid \alpha k\rangle\langle\alpha k \mid a k\rangle \delta\left(\varepsilon-\varepsilon_{\alpha k}\right)
$$

- the projection of the DOS upon the atomic state $a$, as well, familiar from numerous packages (e.g. LOBSTER [40] or WANNIER90 [41]) performing analysis of numerical data derived from PAW-DFT or whatever computer experiments on solids (see e.g. [40, 42]). Treating similarly off-diagonal elements:

$$
G_{a r, b r^{\prime}}(z)=\sum_{\alpha k} \frac{\langle a k \mid \alpha k\rangle\langle\alpha k \mid b k\rangle \exp \left(i k\left(r-r^{\prime}\right)\right)}{z-\varepsilon_{\alpha k}},
$$

yields:

$$
-\frac{1}{\pi \mathrm{K}} \Im G_{a r, b r^{\prime}}(\varepsilon)=\sum_{\alpha k} \Re\left[\langle a k \mid \alpha k\rangle\langle\alpha k \mid b k\rangle \exp \left(i k\left(r-r^{\prime}\right)\right)\right] \delta\left(\varepsilon-\varepsilon_{\alpha k}\right)
$$

which is a close relative of the crystal orbital overlap and crystal orbital Hamilton populations (respectively, COOP and COHP [43]) as well widely available in the solid state packages. In these expressions $|a r\rangle$ corresponds to the $\mathrm{AO} a$ in the unit cell $r$, so, the latter formula allows to calculate the density matrix element for the pair of orbitals from different unit cells.

\section{Green's functions of a finite system}

The definition of the GF and all related quantities in the case of the finite system remains the same as sketched in the Subsection devoted to the GF representation of eigenstates problem. The Greenian matrix element has a general form eq. (5), which for the real values of argument reduces to:

$$
G_{a b}(\varepsilon)=\sum_{\lambda}\left[\frac{\langle a \mid \lambda\rangle\langle\lambda \mid b\rangle}{\varepsilon-\varepsilon_{\lambda}}-i \pi\langle a \mid \lambda\rangle\langle\lambda \mid b\rangle \delta\left(\varepsilon-\varepsilon_{\lambda}\right)\right],
$$

where the summation goes over discrete levels $\lambda$. The imaginary part of the GF consists of discrete signals located at energies $\varepsilon_{\lambda}$ and proportional to the Dirac's $\delta$-"function". The real part is a continuous function except simple poles at $\varepsilon=\varepsilon_{\lambda}$.

In the general case it is not possible to find an analytical solution for the perturbed GF, therefore one has to treat the initial Greenian matrix of the finite system numerically. Such treatment requires to approximate the $\delta$-function in eq. (18) by a Lorentzian of a (small) width $\nu$ as explained in Appendix A. Theoretically, such approximation approaches genuine result in the limit $\nu \rightarrow 0$. In practical calculations the value of this parameter has to be finite. It is advised to set $\nu$ equal to the step of the energy grid and to choose the latter small enough to guarantee required accuracy.

\section{Implementation and Computational Details}

\section{General construction}

The programmatic implementation of the theory described in the Theory Section is provided by GoGreENGo package being a set of procedures written in FORTRAN 2010 using the system of high-level objects provided 
by the CARTESIUS_FORT library [44]. The component procedures exchange data through intermediate files packed in the hdf5 archive [45]. The flow-chart illustrating the relations between different programs of the package and the paths of data transfer between them is shown on the Fig.1. Specifically,

- program GET_GREEN evaluates Greenian matrix elements for a given set of local atomic orbitals from the band structure of the ideal crystal. The program can use eigenvalues derived by VASP [46], ABINIT [47]. The projections of eigenvectors produced by these packages to the basis of local orbitals can be obtained by the LOBSTER software [40] whose output format is compatible with GET_GREEN. As well $\Theta \Phi$ (TetaPhi) [48] produces eigenvalues-eigenvectors in the format readable by GoGreenGo. This combination allows to extract Greenian matrix of the pure solid in an atomic basis from the most popular plane wave DFT codes and use it for impurity calculations. One can use the band structure of the solid from any other source, as well, transforming it to the required format. Imaginary parts of $G_{a b}$ are calculated in GET_GREEN for each point of the energy grid by applying tetrahedron algorithm [49] of integration over the Brillouin zone. Since the real parts of GF elements are related with the imaginary parts by the Kramers-Kronig relations [51] they are obtained numerically from the latter as described in Ref. [52]. The user has to define the interval of the energy, where the Greenian matrix has to be calculated, and the step of the energy grid. In addition, there is an option to obtain elements of the Greenian matrix between different unit cells $r$ and $r^{\prime}$ as in eq.(17) with no calculations on extended unit cells.

- HGEN calculates the crystal-defect hopping operator and an array of two-electron Coulomb integrals (required for the self-consistent setting). Using HGEN is not mandatory: one can also use any externally prepared hopping operator and Coulomb integrals in the required format. HGEN calculates the interaction within a semi-empirical NDDO approximation including different parameterizations such as MNDO [53], AM1 [54] and PM3 [55] which have been recently shown to be compatible with the PAW-DFT setting [56]. Other options of calculating atomic integrals will be added in the future releases. To run the HGEN utility one has to provide geometries of the defect and crystal, define which atoms interact (by giving a cut-off distance or listing them explicitly) and type of the AOs basis set to be used (single STO, MAP [57], Bunge [58], Koga [59] are available so far). Optionally, one can also change default values of semi-empirical hopping parameters in order to parameterize the Hamiltonian for one's needs.

- program DYSON reads unperturbed Greenian matrix of the ideal crystal (which comes from GET_GREEN), that of a defect (also comes from GET_GREEN or can be calculated directly in DYSON if one provides molecular orbital energies and MO LCAO coefficients), initial perturbation operator and a table of two-electronic integrals, if the self-consistent version is used. The program finds a solution of the Dyson equation and returns perturbed Greenian matrix together with the new density matrix and energy correction caused by perturbation. In the case of the self-consistent setting it performs iterative solution of the Dyson's equation taking into account an adjustment of the self-energy at each step. In this case the user has to specify a convergence threshold. The damping ensuring better convergence is supported and can be used if necessary.

- program GREEN_OPT performs either gradient or simplex optimization of the defect position and (internal) geometry. HGEN can be used to generate perturbation matrix on each step. In HGEN no gradients are available so far, so it can only be used for the simplex optimization. Gradients will be added to HGEN in future versions.

\section{Some specific features}

\section{Self-consistent perturbation theory}

As mentioned in the Theory Section, the perturbation of a one-electron part of the Fockian produces a correction to the density matrix of the same order as the perturbation itself. Then, due to the mean field treatment of the electron-electron interaction either in the wave-function or DFT setting, the corresponding Fockian receives further corrections proportional to those of the density matrix elements. They form so called 




Figure 1: Flow-chart of the GoGREEnGo package.

"dressing" which needs to be added to the original one-electron ("bare") perturbation. The solution of the resulting Dyson equation yields further changes of density matrix so that one has to repeat the calculation until the convergence is reached. This option is implemented in the GoGreEnGo package.

In metals the effect of perturbation decays with the distance from the defect as $R^{-2 \kappa}$ with some $\kappa>0$ dependent on the from of the Fermi surface and dimensionality of the crystal structure ( $\kappa$ is typically higher for higher dimension) [27]. ${ }^{6}$ This allows one to restrict the range of the action of the self-consistent (dressed) perturbation by a finite number of unit cells close to the defect. Of course, the corrections to the two-electron part of the Fockian involve additional orbitals, and the dimension of the subspace $P$ has to be increased. The amount of this augmentation is system dependent and it is advised to look for an optimal size by a series of convergence tests.

\section{Position of the Fermi level}

Another important aspect is the position of the Fermi level in the perturbed system. In general, it does not remain constant although changes by a small value. The general reason is that the perturbed GF $\mathbf{G}(\varepsilon)$ differs from unperturbed one $\mathbf{G}^{(0)}(\varepsilon)$ and thus the integral of $\operatorname{SpG}(\varepsilon)$ from $-\infty$ up to $\varepsilon_{F}$ of the unperturbed crystal not necessarily yields the same number of electrons as does the integration of $\operatorname{SpG}^{(0)}(z)$. The defect of electron's number $\Delta n$ is to be eliminated by shifting the Fermi energy by $\delta \varepsilon_{F}$. As it is explained in Appendix $\mathrm{C}$ the value of $\delta \varepsilon_{F}$ is determined by the value of $\Delta n / \mathrm{K}$ and it, evidently, becomes infinitesimally small for the limit $\mathrm{K} \rightarrow \infty$. In real calculations $\mathrm{K}$ is finite and $\delta \varepsilon_{F}$ is non-vanishing in this case. Evaluation of $\delta \varepsilon_{F}$ required to keep constant the number of electrons in the system with $\mathrm{K}$ unit cells is implemented in the GoGreenGo. Practically, in most our calculations the value of $\Delta n / \mathrm{K}$ is rather small (we use $31 \times 31 \times 31$ meshes of $k$-points for three-dimensional models and $51 \times 51$ one for graphene) and affects density matrix elements only in fourth-fifth decimal place. However, in all cases it is advised to thoroughly check its impact on the density matrix elements and final results, especially if the number of $k$-points $\mathrm{K}$ used for the band structure calculations of the crystal is relatively small as it sometimes happens in PAW-DFT calculations.

\footnotetext{
${ }^{6}$ This is the most unfavorable situation - the decay in insulators is even faster.
} 


\section{Energy correction}

Even an infinitesimally small shift of the Fermi level causes a finite correction to the perturbation energy, since a summation over infinite number of unit cells is implied in calculations. It may be shown [28] that the perturbation energy is:

$$
\delta E=-\frac{1}{\pi} \int_{-\infty}^{\varepsilon_{F}}\left(\varepsilon-\varepsilon_{F}\right) \delta \Im \operatorname{SpG}(\varepsilon) d \varepsilon=-\frac{1}{\pi} \int_{-\infty}^{\varepsilon_{F}} \varepsilon \delta \Im \operatorname{Sp} \mathbf{G}(\varepsilon) d \varepsilon-\Delta n \varepsilon_{F},
$$

where $\Delta n$ is a difference in number of electrons in the perturbed system (calculated with the original Fermi level of the unperturbed system) and the initial one. In the self-consistent version of the calculations the modified equation [24]:

$$
\begin{gathered}
\delta E=-\frac{1}{\pi} \int_{-\infty}^{\varepsilon_{F}}\left(\varepsilon-\varepsilon_{F}\right) \delta \Im \operatorname{Sp} \mathbf{G}(\varepsilon) d \varepsilon- \\
-\frac{1}{2} \sum_{i, j, k . l}\left(P_{i j} P_{k l}(i j|| k l)-\left(P_{i j}^{(0)}\right)_{0}\left(P_{k l}^{(0)}\right)_{0}(i j|| k l)_{0}\right)
\end{gathered}
$$

taking into account changes in the self-energy needs to be used. The summation in the last term goes over all orbitals involved in the perturbed subspace $P$. Due to the decaying effect of the perturbation, it is a finite set as explained above. Two-center two-electronic integrals in this equation change in the perturbed system only if the geometry gets distorted.

\section{Local and virtual states}

As it was stressed yet in works $[11,19]$, the defects may produce additional poles of the perturbed GF of two types. Either so-called local or virtual states may arise depending on the strength of the perturbation as related to the energy spectrum of the unperturbed system. The local states correspond to the poles on the real axis and, therefore, appear as narrow peaks of the perturbed DOS in the energy ranges, where the unperturbed DOS vanishes - outside the allowed energy bands of the ideal crystal. By contrast, the virtual states are related with the poles in the complex plane [18] and manifest themselves on the real axis as wide Lorentzian peaks of the perturbed DOS inside the allowed energy band (see Appendix D). Both features are perfectly reproduced by the GoGREENGo package as demonstrated in Figs. 4, 6. For more details about handling the pole structure of the perturbed GF in GoGreEnGo see Appendix D.

\section{Test Results and Discussion}

Since the described approach, although, well established theoretically (analytically), did not so far enjoy full scale program implementation, it requires a thorough testing against analytically solvable models, even looking out oversimplified. Below, we present such tests and round up with an intermediate test - adsorption on graphene, which on one hand can be traced analytically far enough to provide necessary reference and on the other hand provides, although a simple, but realistic, example eventually suitable for experimental check. In the main text we mostly concentrate on the numerical results obtained by GoGREENGo, while analytical solutions, used for control, are collected in the Supporting Materials. Even in the case of simple models not all functionality of the package can be tested against analytical solutions, since the later are available only for the simplest local perturbations and are inaccessible in the frame of the self-consistent approach. Therefore, we test GoGreEnGo against very simple benchmarks and then demonstrate its capabilities for more realistic and comprehensive setting.

\section{GoGreenGo for perturbation of cubia}

Cubia (see e.g. Ref.[60]) are simplest thinkable models of 3D metals. They are formed by $s$-orbitals centered at the vertices of (simple - sc, body centered - bcc, and face centered - fcc) cubic lattices with one-electron hopping $t$ between the nearest neighbors of a given node of a lattice. The dispersion laws of electronic bands for such models [61] allowing for analytic solutions as given in Table 1. Correspondingly, the eigenvectors 
Table 1: Dispersion relations, Fermi level and the nearest neighbor Coulson bond-order for cubia with single (one electron per site) occupation. Indices $k_{i}$ correspond to projection of wave vector $k$ to orthogonal basis vectors of reciprocal space chosen so that in all cases the cubic Brillouin zone is defined as $-\pi<k_{\kappa} \leq \pi$.

\begin{tabular}{|c|c|c|c|c|}
\hline & $\varepsilon_{k}$ & $W$ & $\varepsilon_{F}$ & $B$ \\
\hline sc & $-2 t\left(\cos k_{x}+\cos k_{y}+\cos k_{z}\right)$ & $12 t$ & 0 & 0.3324 \\
\hline bcc & $-8 t \cos k_{x} \cos k_{y} \cos k_{z}$ & $16 t$ & 0 & 0.2605 \\
\hline fcc & $-4 t\left(\cos k_{x} \cos k_{y}+\cos k_{x} \cos k_{z}+\cos k_{y} \cos k_{z}\right)$ & $16 t$ & $0.915 t$ & 0.2184 \\
\hline
\end{tabular}

(Bloch states) related to these eigenvalues are known and, consequently, the Green's functions. Specifically, diagonal GFs for the systems listed in Table 1 are known from Refs. [61,62,63,64] and their plots are presented in Supporting Material Sec. 1. One can also find the corresponding graphs of the electronic DOS at the website [65]. The off-diagonal elements of the respective Greenian matrices are as well accessible through GoGreenGo.

It is as well possible to find analytical solutions for the ideal cubia in the framework of the extended Hubbard model taking into account electron-electron interactions as described in Appendix E. In our subsequent consideration we will use the extended Hubbard model as a starting point for the self-consistent calculations. All energy parameters are given in units of $t$, that is $t=1$ everywhere below. The diagonal matrix elements of the unperturbed Fockian are set to zero being by this the energy reference. In further Subsections we present as coherence tests the results of numerical treatment of various local perturbations of cubia with use of GoGreEnGo package.

\section{Lattice substitutions in cubia}

First, we consider lattice substitution defects in cubia, where one atom of the crystal (denoted as "0") is replaced by a different atom. In general, the substitute can be a many-electron atom, but here we restrict our tests by single-electron impurities. Chemically this corresponds to substitution defects in alkali metals.

Complexity of the model can be gradually increased by including different terms into the perturbation operator so that different program features are tested independently. In the simplest possible setting we neglect electron-electron interactions and only take into account one-center perturbation: the difference $v$ between diagonal matrix elements of the Fockian over impurity AO and the AO's of the unperturbed crystal. In this case an analytical solution is available (Supporting Material Sec. 3). Comparing numerical results to this solution shows that GoGreEnGo produces the perturbed Greenian matrix identical to the analytical one up to eighth decimal sign. In addition, this setting allows to make qualitative sketch of the features of the perturbed system, which remain valid for more involved crystals and perturbations of this kind. As one can conclude from the analytical form of the mass operator (see Appendix D) and of the cubia GFs $G_{00}^{(0)}$ (Supporting Material Sec. 1), the additional poles in the perturbed system appear for: (i) sc $|v|>4.01$; (ii) bcc $|v|>6.10$; (iii) fcc $v<-9.38$ in the case of occupied local state and $v>0.83$ in the case of vacant local state. In all these cases except for the local state above the band in the fcc lattice, the required value of $v$ is unreasonably high and it is difficult to expect any local state to appear if, say, indeed, one alkali atom is exchanged by another (the typical difference in core attractions lies in the range of 0.4-0.8 eV [66] - much smaller than the typical bandwidth). The only option is an appearance of the vacant local state in the fcc lattice, but it presents a minor interest since it does not contribute to the electronic density and the energy of the system. Another conclusion, which can be drawn from the analytical solution, is the behavior of the perturbed function in the vicinity of the pole of the initial GF in bcc. For bcc $\Im G_{00}^{(0)}$ is even function of $\varepsilon$ and $\Re G_{00}^{(0)}$ is odd. In addition, due to the presence of the pole at $\varepsilon=0, \Re G_{00}^{(0)}$ has a discontinuity there. Therefore, one would observe a discontinuity of the perturbed function $\operatorname{DOS}_{0}(\varepsilon)$ at $\varepsilon=0$, which is indeed observed in our numerical results described below (see Fig. 2).

Although described model provides a simple and pictorial solution, it is rather far from realistic description of the substitution defects, requiring more involved interaction operator and the self-energy corrections. GoGreenGo supports such description and to test this, we included corrections to one-center two-electron and two-center hopping integrals, Coulomb interactions, and applied the self-consistent procedure. We performed calculations within this setting for cubia lattices for $v= \pm 1(t)$ complemented by a set of further 
Table 2: Parameters of charge distribution in cubia with the substitutional defect and electronic energy of the defect formation. $Q_{i}=1-n_{i}$ corresponds to the atomic charge of $i$-th neighbor of the defect site "0" with $n_{i}$ being the electronic population of the $i$-th site in the perturbed system.

\begin{tabular}{|c|c|c|c|c|c|c|c|c|}
\hline & $v$ & $Q_{0}$ & $Q_{1}$ & $Q_{2}$ & $Q_{3}$ & $\sum_{i \sim j} \delta B_{i j}$ & $\Delta n$ & $\delta E$ \\
\hline \multirow{2}{*}{ sc } & -1 & -0.3478 & 0.1216 & -0.0712 & 0.0555 & -0.1620 & 0.0882 & -1.9964 \\
\cline { 2 - 9 } & 1 & 0.3478 & -0.1217 & 0.0711 & -0.0555 & -0.1594 & -0.0899 & -0.0029 \\
\hline \multirow{2}{*}{ bcc } & -1 & -0.4067 & 0.1058 & -0.0835 & -0.0389 & -0.0600 & 0.1488 & -2.0900 \\
\cline { 2 - 9 } & 1 & 0.4043 & -0.1088 & 0.0819 & 0.0379 & -0.0592 & -0.0121 & -0.0866 \\
\hline \multirow{2}{*}{ fcc } & -1 & -0.1984 & 0.0243 & -0.0115 & -0.0025 & -0.0532 & 0.0294 & -2.1583 \\
\cline { 2 - 9 } & 1 & 0.2903 & -0.0293 & 0.0116 & 0.0025 & -0.0413 & -0.0766 & -0.2671 \\
\hline
\end{tabular}

parameters: variation of the hopping between the impurity and its neighbors ( $\delta t=-0.2)$, one-center Coulomb repulsion in the unperturbed system $\left(\gamma_{0}=0.6\right)$, two-center (nearest neighbor) Coulomb repulsion in the unperturbed system $\left(\gamma_{1}=0.3\right)$ and a variation of the Coulomb integrals in the defect $\left(\delta \gamma_{0}=-0.1\right.$ and $\left.\delta \gamma_{1}=0.1\right)$. The resulting perturbed $\operatorname{DOS}_{0}(\varepsilon)$ together with obtained charge distributions are depicted in Fig.2; numerical values of charges and the electronic ${ }^{7}$ energy variations due to the defect formation are collected in Table 2.

In all cases the charge distributions induced by the defect have a similar oscillating-decaying behavior. The defect site ("0") carries the highest (by absolute value) net-charge, negative (electron density accumulation) for $v=-1$ and positive (electron density depletion) for $v=1$. Absolute values of the net-charges on other sites decay with the separation from the defect and have alternating signs so that for any site its charge has the opposite sign to the charges of its neighbors. The decay rate is rather high as expected for 3D metals [27]. The fastest one is observed for fcc, where the induced charges are insignificant $(<0.01)$ beyond the $2^{\text {nd }}$ neighbors. For bcc the corrections become negligible beyond $3^{\text {rd }}$ neighbors and for sc beyond the $4^{\text {th }}$ ones. In all cases the Fermi level shifts as described in the Implementation Section and Appendix C, but the absolute values of $\delta \varepsilon_{F}$ do not exceed $7 \cdot 10^{-6}$. For $v=-1$ it shifts downwards and for $v=1$ - upwards. Such a small shift insignificantly affects individual density matrix elements. Maximal correction of the diagonal density matrix elements due to the Fermi level shift equals to $\Delta n / \mathrm{K}$.For $31 \times 31 \times 31 k$-mesh used in our calculations and values of $\Delta n$ from Table 2 they never exceed $5 \cdot 10^{-5}$ for cubia, which is fairly negligible.

The (Coulson) bond orders - the off-diagonal elements of the one-electron density matrix - are affected much less than the diagonal matrix elements of the density. Again, these corrections decay rapidly with the distance from the defect. In all cases, the bonds formed by the defect site are weaker than those of the innate atom, but even this correction occurs only at the third decimal place. Nevertheless, the sum of all corrections to the bond orders $\sum_{i \sim j} \delta B_{i j}$ can be fairly noticeable due to the large number of bonds in the cubia lattices. These values are presented in Table 2 . As one can see, in all cases the total variation of the bond orders is negative, meaning that the system loses a part of the bonding energy because of the defect.

Electronic energy of the defect formation can be evaluated by eq. (20). An alternative expression for it is given in Appendix E. As it can be seen from Table 2, in all cases the total energies are negative and the absolute values are much higher for the systems with $v=-1$, which is obviously explained by the sign of the one-center contribution of the impurity AO. Further significant contributions are the Coulomb attraction of the atomic charges, which is always negative due to the oscillatory behavior of the charge distribution, and the correction to the bonding energy, which is always positive as we saw above.

\section{Substitution defects in $p$-cubium}

In the previous Subsection, we have tested the main functionality of GoGREENGo for cubia - single-band crystals. To demonstrate package's capabilities in treating multi-band solids we use a $p$-cubium (pc) model, which is formed by three $p$-orbitals located in the vertices of the simple cubic lattice. Due to symmetry, each orbital overlaps only with its own nearest translation images in the frame of the tight-binding approximation (e.g. $p_{x}$ orbital overlaps only with neighboring $p_{x}$ orbitals and orthogonal $p_{y}$ and $p_{z}$ ). Two of such overlaps correspond to $\sigma-\sigma$ interaction and four others to $\pi-\pi$ interaction. In addition we assume the following for

\footnotetext{
${ }^{7}$ Correction to core-core repulsion term caused by the defect is not included.
} 


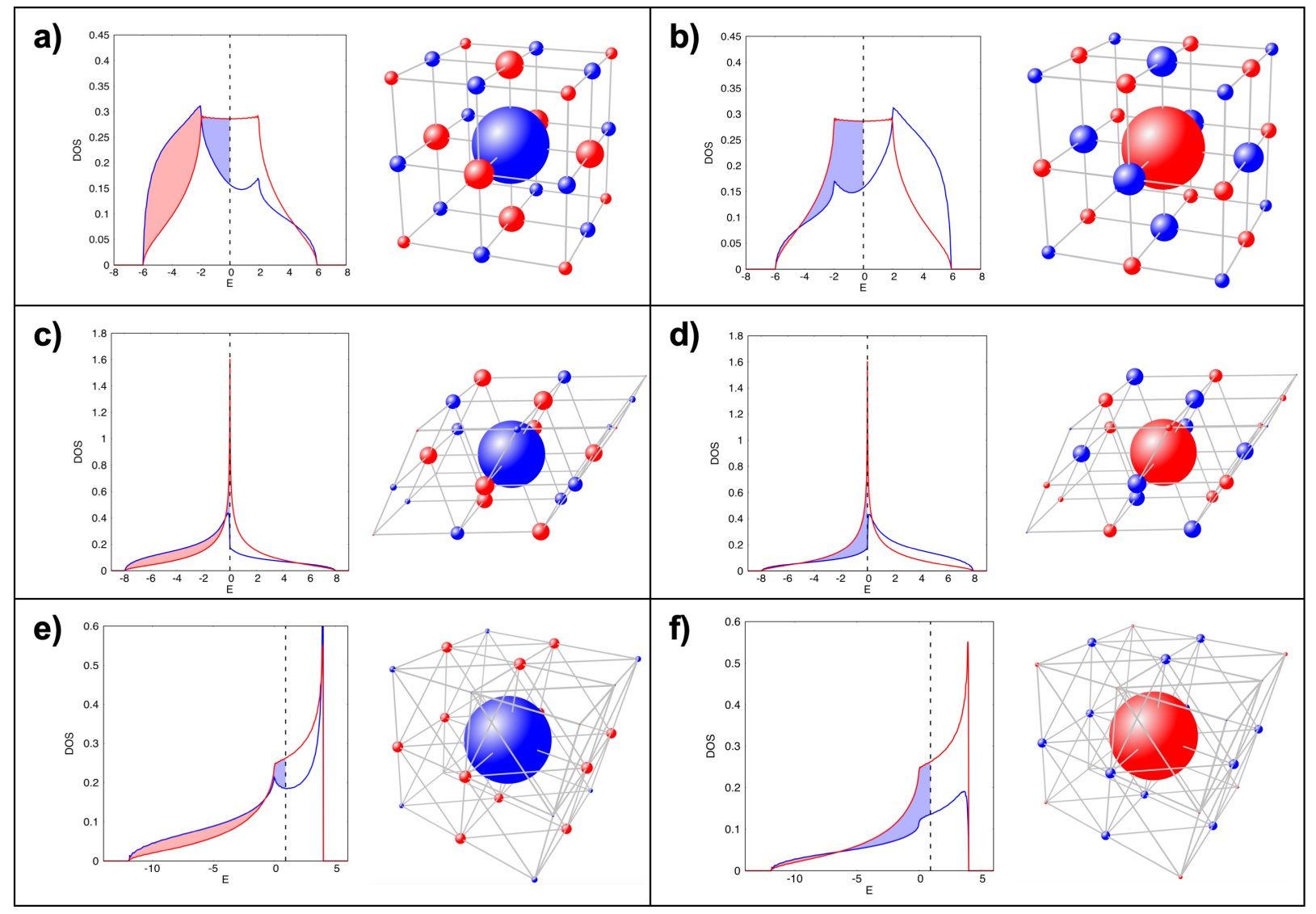

Figure 2: Perturbed densities of states $\operatorname{DOS}_{0}(\varepsilon)$ (blue lines) in comparison with the initial ones (red lines) for the cubia lattices and schematic representations of atomic charges distributions induced by the substitutional defects. Dashed vertical lines show the Fermi level. Individual panels correspond to the following systems: a) sc $v=-1$; b) sc $v=1$; c) bcc $v=-1$; d) bcc $v=1$; e) fcc $v=-1$; f) fcc $v=1$. Radii of the spheres are proportional to the charge of the atom (notice the different scales of $Q_{0}$ for different lattices in Table 2 ). Red spheres correspond to the positive charge, blue - to the negative. The biggest sphere in all cases is located on the defect site ("0"). On the plots red filling between the curves corresponds to the increase of the diagonal electronic density on the site and blue one - to decrease. 
the two-electron Coulomb integrals $(a b \mid c d)=(a a \mid c c) \delta_{a b} \delta_{c d}$, which allows to keep the same number of twoelectron parameters as previously. The analytical band-structure for the ideal pc contains three degenerate bands (see e.g. Ref. [60]):

$$
\varepsilon_{\alpha k}=-2 t\left(-\cos k_{\alpha}+\kappa \sum_{\beta \neq \alpha} \cos k_{\beta}\right)
$$

where $\alpha, \beta=x, y, z$ numerates bands and projections of $k$-vector, $\kappa$ is a ratio between $\pi-\pi$ and $\sigma$ - $\sigma$ hoping parameters and $t$ stands for the $\sigma-\sigma$ one. Minus in front of the first cosine in the brackets occurs because the $\sigma-\sigma$ overlap of two $p$-orbitals is negative (and hopping is positive), if they are aligned in the same direction. In our further consideration we set $\kappa=0.40$ and $t=1$. The elements of the Greenian matrix for pc are accessible through GoGreenGo and can be found in Supporting Material Sec. 1. We consider pc with one electron per unit cell, yielding the Fermi level to be $\varepsilon_{F}=-1.753 t$. Coulson bond-order $B^{\sigma}$ for the $\sigma$-bond is -0.2753 and for the $\pi$-bond is $B^{\pi}=0.1474$. Note, that $B^{\sigma}$ is negative and being combined with positive $\sigma-\sigma$ hopping produces a negative contribution to the energy. In our further discussion of overall change in the bond orders it is convenient, therefore, to use values of $\left|B^{\sigma}\right|$.

To test the whole functionality of the program in the case of multi-band crystal we consider substitutional defects in the most rigorous setting by taking into account the self-energy correction and changes of one- and two-center integrals as it was done in the previous Subsection. The set of parameters used for calculations: $v= \pm 0.5, \delta t=-0.2, \gamma_{0}=0.6, \gamma_{1}=0.3, \delta \gamma_{0}=-0.1$ and $\delta \gamma_{1}=0.1$.

Perturbed DOS's projected on one of the $p$-orbitals of the defect site "0" are plotted on Fig. 3 for the negative and positive perturbation. Resulting charge distributions exhibit similar behavior as in sc with some specific variations. First of all, the charges decay faster than in sc: they become negligible beyond 3rd neighbors for $v=-0.5$ and beyond 4th for $v=0.5$. Moreover, charge signs follow a different pattern: (i) for $v=-0.5$ the charges are $Q_{0}=-0.4152, Q_{1}=0.0195, Q_{2}=0.0102, Q_{3}=-0.0042$; (ii) for $v=0.5$ they are $Q_{0}=0.3039, Q_{1}=-0.0245, Q_{2}=-0.0044, Q_{3}=0.0217$. That is, the charges change their signs every two neighbors from the defect. Also, it can be seen that the negative perturbation causes a larger charge than the positive one of the same magnitude. This is a consequence of a nonsymmetric location of the Fermi level in the band (like in fcc cubium). Values of $\Delta n$ are 0.1523 for the negative perturbation and -0.2524 for the positive one. As previously, such small values do not cause significant shift of the Fermi level for our $31 \times 31 \times 31 k$-mesh.

As for corrections to the bond orders, we see that they change significantly only for the bonds incident to the " 0 " site ( $\sigma$-bond becomes weaker and $\pi$-one stronger in all cases). The total changes in bond orders are $\sum_{i \sim j} \delta B_{i j}=-0.1541$ for the negative perturbation and $\sum_{i \sim j} \delta B_{i j}=-0.2414$ for the positive perturbation, so the system loses bonding energy, upon formation of the defect.

\section{One-orbital interstitial impurity in simple cubium}

Further class of problems accessible for GoGREENGo is that of interactions of solids with some extra additive termed as an interstitial defect. The simplest example of such defect is an interstitial (impurity) atom represented by a single $s$-orbital, which may be considered as a model of a hydrogen atom. We considered the effect of such impurity placed in a cubic void of the sc lattice being a nontrivial example illustrating general features of the methodology. In the cubic void, the defect is surrounded by eight identical atoms of the crystal system. The unperturbed Fockian of such a system is one of the crystal augmented by an extra row and column having the energy of the impurity orbital on their intersection and filled by off-diagonal zeroes signifying no interaction between the impurity and the crystal. The perturbation consists, in the first approximation, of one-electron hopping between the defect $(s$-)orbital and those of its immediate neighbors in the lattice (eight neighbors). These nine orbitals form the perturbation subspace (the $P$-subspace of eq. (11) ) hereinafter addressed as the "impurity cluster".

The position of the defect $s$-orbital relative to the cubium Fermi level is given by a difference between ionization potentials of the interstitial atom and crystal atom of the lattice. For all metals, atomic ionization potentials are smaller than for hydrogen, therefore we assume that the diagonal matrix element over the impurity orbital is negative. In the cubia models the nearest neighbor hopping parameter $t$ of the crystal provides the natural energy scale. We, at first, considered numerically the effect of interaction on the atomic 


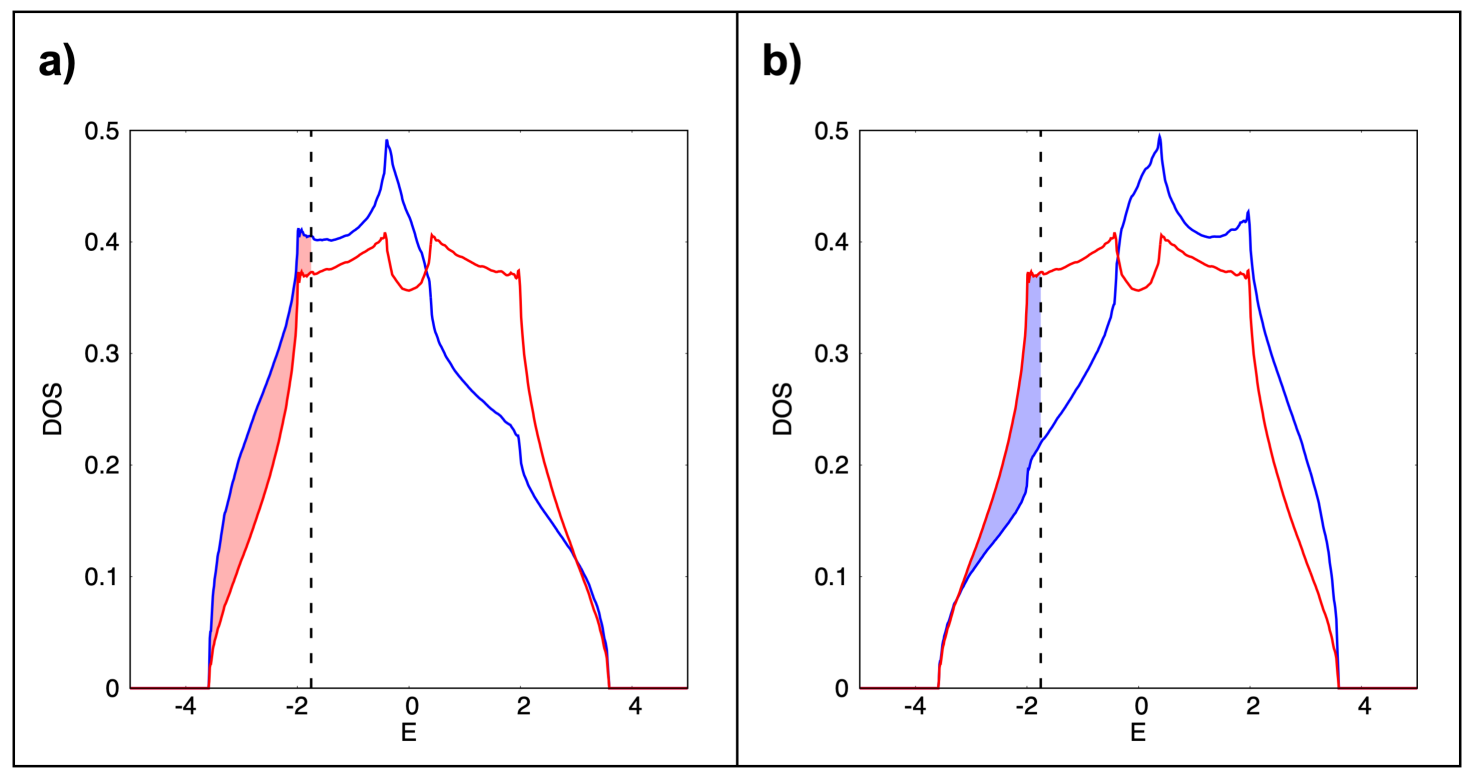

Figure 3: Perturbed densities of states $\operatorname{DOS}_{0}(\varepsilon)$ (blue lines) compared with the ideal ones (red lines) for the substitution defect in $p$-cubium : a) $v=-0.5$; b) $v=0.5$. Color scheme is the same as in Figure 2 .

Table 3: GoGreenGo results obtained for the hydrogen impurity atom in the cubic void with the Anderson Hamiltonian (Supporting Material Sec. 3.2). Parameter $\gamma=0.40$ for all cases.

\begin{tabular}{|c|c|c|c|c|c|c|c|c|}
\hline$\varepsilon_{a}$ & \multicolumn{5}{|c|}{-2} & \multicolumn{3}{|c|}{0} \\
\hline$t^{\prime}$ & 0.20 & 0.70 & 0.83 & 0.95 & 0.20 & 0.70 & 0.83 & 0.95 \\
\hline$n_{a}$ & 1.9910 & 1.6823 & 1.4164 & 1.2164 & 0.6872 & 0.6023 & 0.6319 & 0.6557 \\
\hline$n_{i}$ & 0.9987 & 0.9608 & 0.9347 & 0.9130 & 0.9887 & 0.9534 & 0.9474 & 0.9418 \\
\hline$B$ & 0.3311 & 0.2932 & 0.2672 & 0.2454 & 0.3211 & 0.2858 & 0.2798 & 0.2742 \\
\hline$B^{\prime}$ & 0.0108 & 0.1286 & 0.2072 & 0.2446 & 0.1381 & 0.2772 & 0.2909 & 0.2995 \\
\hline$\delta E$ & -1.8278 & -2.2008 & -2.3433 & -2.0104 & -0.2086 & -2.0233 & -2.6101 & -3.0670 \\
\hline
\end{tabular}

charges and bond orders in the "impurity cluster" in the frame of the Anderson model (see Supporting Material Sec. 3.2). Analytical solutions for the Anderson model used for testing are given in Supporting Material Sec. 3.2.

Perturbed GFs obtained numerically with GoGREEnGo for a "hydrogen" atom in a cubic void (for the first step of the self-consistent procedure) were compared with corresponding analytical equations from Supporting Material Sec. 3.2. The values coincide up to $6^{\text {th }}$ decimal place. Corrections to density matrix elements are not accessible analytically due to a very involved functional form of $\mathbf{G}^{(0)}$ even for the sc cubium. Numerical values are collected in the Table 3 . As one can conclude, if the adsorbate level is positioned below the Fermi level the impurity atom acquires a negative charge, while for $\varepsilon_{a}=\varepsilon_{F}=0$ the charge is positive. The absolute value of charge depends on the impurity-matrix hopping parameter $t^{\prime}$ - as it increases, the charge decays which is a result of changing in coupling patterns (see the four types of perturbed adsorbate levels in Supporting Material Sec. 3.2.). Atoms of the crystal matrix in all cases acquire a small positive charge.

Bond orders $B$ between the crystal atoms of the "impurity cluster" become smaller (the bonds become weaker) and this energetically unfavorable change is compensated by formation of new bonds with the order $B^{\prime}$ between impurity atom and lattice atoms. With increasing hopping parameter $t^{\prime}$ the orders of new bonds increase and the orders of original bonds decrease. For $\varepsilon_{a}=0$ the bond orders $B^{\prime}$ are larger than for $\varepsilon_{a}=-2$.

In a more extended setting, we turn on the Coulomb interactions in the crystal. In this case, non-vanishing 
Table 4: GoGreenGo results obtained for the hydrogen impurity atom in the cubic void with taking into account inter-electronic interactions in the crystal. Parameters $\gamma=\gamma_{0}=0.4, \gamma_{1}=\gamma^{\prime}=0.2$ for all cases (see Supporting Material Sec. 3.2 for the equations).

\begin{tabular}{|c|c|c|c|c|c|c|c|c|}
\hline$\varepsilon_{a}$ & \multicolumn{5}{|c|}{-2} & \multicolumn{3}{c|}{0} \\
\hline$t^{\prime}$ & 0.20 & 0.70 & 0.83 & 0.95 & 0.20 & 0.70 & 0.83 & 0.95 \\
\hline$n_{a}$ & 1.9914 & 1.6814 & 1.4592 & 1.2321 & 0.6242 & 0.6079 & 0.6387 & 0.6654 \\
\hline$n_{i}$ & 0.9470 & 0.9306 & 0.9330 & 0.8935 & 1.0098 & 0.9816 & 0.9748 & 0.9701 \\
\hline$B$ & 0.3296 & 0.2887 & 0.2779 & 0.2313 & 0.3198 & 0.2833 & 0.2770 & 0.2728 \\
\hline$B^{\prime}$ & 0.0116 & 0.1336 & 0.2417 & 0.2511 & 0.1450 & 0.2791 & 0.2923 & 0.3027 \\
\hline$\delta E$ & -1.8720 & -2.2704 & -4.5067 & -2.0715 & -0.2247 & -2.0645 & -2.6345 & -3.3018 \\
\hline
\end{tabular}

matrix elements between the sites outside the impurity cluster appear, which makes an analytical treatment unfeasible. The point to be checked here, is the extension of the number of atoms in the impurity cluster for accounting of the perturbation dressing through electron-electron interactions. According to the tests, it is sufficient to include first and second neighbors of the lattice atoms directly interacting with impurity.

As it can be concluded from Table 4 including two-electron Coulomb terms and the self-energy correction does not have major influence on the resulting density matrices and energies; all trends remain fairly the same with slight numerical modifications. The only exception is the case of $\varepsilon_{a}=-2$ and $t=0.83$, where one observes more significant differences in density matrix elements $n_{a}, B$ and $B^{\prime}$ between two settings, which translates into quite considerable difference in energies.

\section{GoGREENGo for perturbations of graphene}

Two-dimensional graphene sheets are widely studied experimentally and theoretically being nature models for two dimensional solids and, respectively, surfaces. Either lattice impurities or adsorbates in/on graphene are of considerable interest from the experimental and theoretical points of view [67, 68].

The honeycomb lattice of graphene has a primitive cell containing two carbon atoms. The $\pi$-system is formed by $p_{\pi}$-orbitals, one for each site of the lattice. Dispersion law in the approximation of the nearest neighbor hopping is:

$$
\varepsilon(k)= \pm t \sqrt{3+2 \cos k_{x}+2 \cos k_{x}+2 \cos \left(k_{x}-k_{y}\right)}
$$

where " -" corresponds to the filled band and "+" to the empty one. Eq. (22) allows analytical evaluation of the diagonal DOS [69] (see also Supporting Material Sec.2 ). The Fermi level $\varepsilon_{F}=0$ and the Coulson bond order $B=0.525$ between nearest neighbors.

In the present Section the Greenian matrix of pure graphene serves as a starting point for the study of several types of local defects. We concentrate on the effect of the local perturbations on the $\pi$-system of graphene and for the time being ignore the $\sigma$-system.

\section{Substitution defects in graphene}

Substitution defects in graphene can be described in the same manner as in cubia. Here we aim to study defects, which are closer to the experimental situation. Specifically, we consider the graphene layer, where one of the carbon atoms is replaced by nitrogen or boron. This is modeled by an appropriate choice of parameters describing one- and two-center interactions as described in Supporting Material Sec. 4. The parameters used to study boron and nitrogen impurities in graphene lattice are collected in Table 5.

The bare perturbed subspace is spanned by four $\pi$-orbitals, one of boron/nitrogen and three more of their nearest neighbors. The perturbation itself touches the diagonal matrix element of the substituted atom and the hopping integrals with its nearest neighbors.

For 2D graphene being a poor metal (Fermi surface degenerates to two - Dirac - points ) one can expect a slower decay of perturbation effect with the separation from the defect. To ensure we do not miss any significant corrections to the density matrix elements we performed self-consistent calculations of substitutional defects using an impurity cluster formed by $7 \times 7$ original cells, whose unperturbed Greenian 
Table 5: Atomic parameters used in this work to describe boron and nitrogen impurities in graphene lattice. All values are in units of $t$.

\begin{tabular}{|c|c|c|c|c|}
\hline & $v$ & $\beta$ & $\gamma_{0}$ & $\gamma_{1}$ \\
\hline $\mathrm{C}$ & - & 0.792 & 2.100 & 0.792 \\
\hline $\mathrm{B}$ & 2.488 & 0.914 & 1.228 & 0.699 \\
\hline $\mathrm{N}$ & -1.251 & 0.904 & 3.573 & 0.862 \\
\hline
\end{tabular}

Table 6: Parameters of charge distribution in the graphene with the substitutional defects and electronic energy of the defect formation. $Q_{i}$ corresponds to the atomic charge of $i$-th neighbor of the defect site "0".

\begin{tabular}{|c|c|c|c|c|c|c|c|c|c|c|}
\hline & $Q_{0}$ & $Q_{1}$ & $Q_{2}$ & $Q_{3}$ & $Q_{4}$ & $Q_{5}$ & $Q_{6}$ & $\sum_{i \sim j} \delta B_{i j}$ & $\Delta n$ & $\delta E$ \\
\hline B & 0.74 & -0.39 & 0.24 & -0.22 & -0.16 & 0.14 & -0.09 & -0.76 & -1.10 & 1.04 \\
\hline $\mathrm{N}$ & -0.42 & 0.27 & -0.17 & 0.15 & 0.12 & -0.10 & 0.07 & -0.42 & 0.91 & -1.42 \\
\hline
\end{tabular}

matrix was derived from the band structure of the unit cell with two atoms eq. (22). As previously we denote the defect site as " 0 ".

Diagonal DOS on the "0"-th site together with schematic representation of the charge distribution in the perturbed systems is given in Fig. 4. The charge distribution parameters and electronic energies of the defect formation are collected in Table 6 . As one can see from the plots of $\operatorname{DOS}_{0}(\varepsilon)$ the local states outside the band appear, respectively, below the band for nitrogen $\left(\varepsilon_{l}=-3.053\right)$ and above it for boron $\left(\varepsilon_{l}=3.773\right)$. For nitrogen the local state contributes 0.38 to the diagonal density matrix element which is $26.95 \%$ of the total value. In the case of boron the local state is vacant and does not contribute. The Fermi level in both cases shifts since nitrogen brings one more electron to the system (hence $\Delta n=0.91$ ) and boron withdraws one electron (hence $\Delta n=-1.10$ ). However, the value of $\Delta n / \mathrm{K}$ is again very small and does not affect the individual density matrix elements significantly (we used $51 \times 51 k$-mesh ).

The qualitative behavior of the atomic charge distribution is fairly similar to that observed in cubia with the only exception that values of the charges decay much slower here, as anticipated. In both cases the corrections to density become negligible $(<0.01)$ beyond $6^{\text {th }}$ neighbor. This is rather reasonable response to very strong perturbation induced by substitutions. Spectacular alternation of signs of the charges induced by substitution seen in Figure 4 is nothing, but the manifestation of the Coulson's "law of alternating polarity" [70], which had been known already to Hückel [71] and served to explain the rules of ortho-meta-para orientation in the electrophilic substitution in benzene derivatives known in organic chemistry [72] .

In complete analogy with cubia, the bond orders are much less affected by the predominantly diagonal perturbation. This effect manifests only for the bonds closest to the defect and become negligible as from the bonds between $3^{\text {rd }}$ and $4^{\text {th }}$ neighbors. The order of the bond closest to the defect decreases by 0.048 for nitrogen and 0.165 for boron. The total change in bond orders $\sum_{i \sim j} \delta B_{i j}$ is, however, quite significant due to the number of bonds involved (see Table 6) and comprises the contributions of the bond orders up to those between $3^{\text {rd }}$ and $4^{\text {th }}$ neighbors, therefore being more local than the variation of diagonal densities (charges).

Energies of the defect formation have different signs - negative for nitrogen and positive for boron. In the latter case this is the result of the huge positive one-center contribution.

\section{Vacancy in graphene}

Vacancies in graphene lattice are interesting from two viewpoints. First, they can appear in the material during synthesis and, therefore, can have an impact on the properties of available samples. Second, vacancies in the $\pi$-system are tentative models of a carbon atom forming an extra bond with some unsaturated particle, which acquiring the $\mathrm{sp}^{3}$ hybridization, leaves the $\pi$-system. In the case of not self-consistent setting analytical GFs can be found, which are given in Supporting Materials Sec. 4. Comparison of GoGreenGo numerical GFs to analytical ones showed they coincide up to eight decimal place. To study vacancy in a more realistic setting we again apply the self-consistent option of the package with one- and two-center parameters of carbon being the same as in the previous Subection.

As before the perturbation cluster involves four orbitals - one excluded from the $\pi$-system and three its 


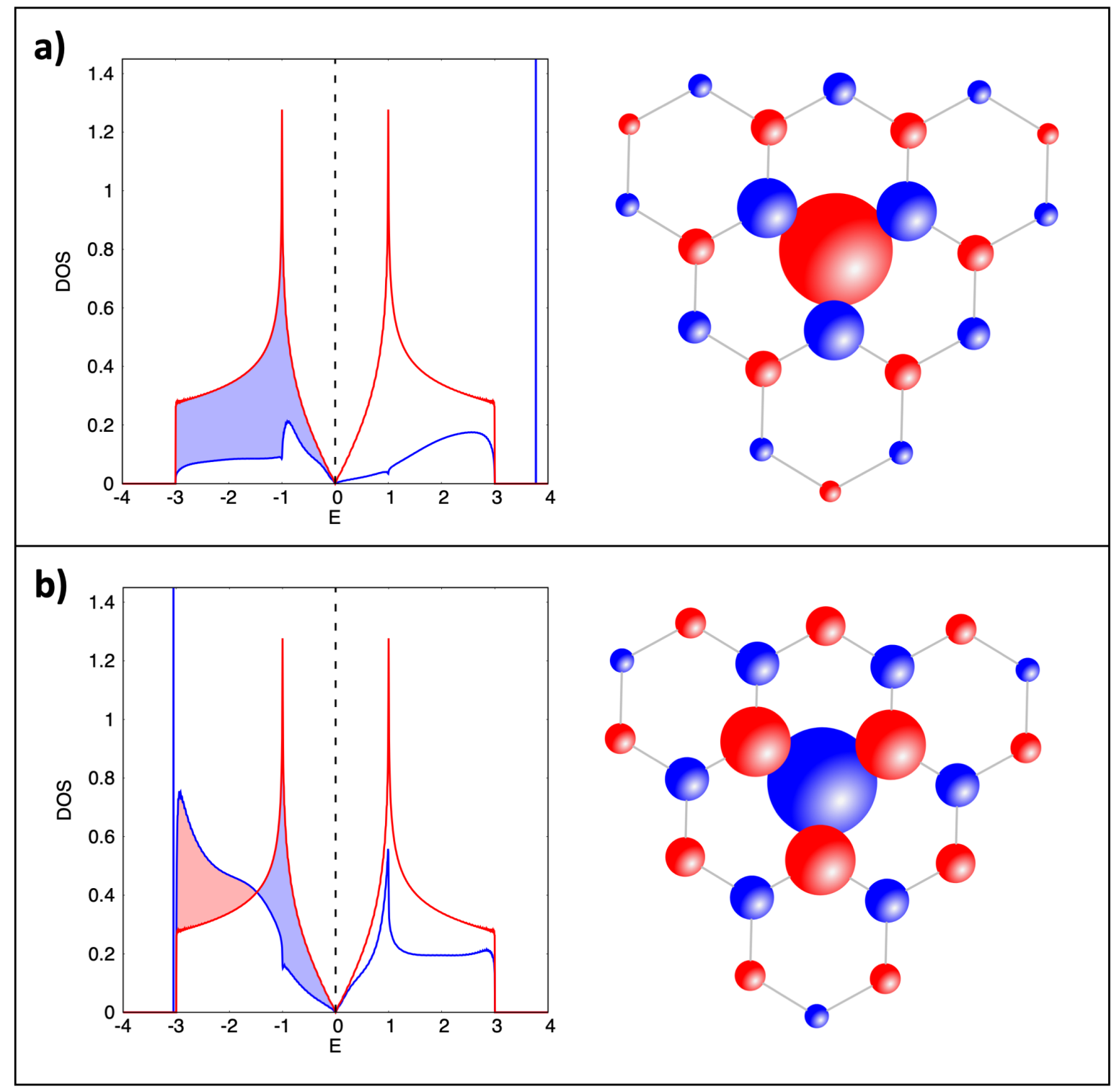

Figure 4: Perturbed diagonal densities of states $\operatorname{DOS}_{0}(\varepsilon)$ in comparison with the initial ones and schematic representation of the charge distributions in graphene with the substitution defects: a) boron, b) nitrogen. Color code and other legend is the same as in Fig. 2. Narrow peaks on the plots above the band in panel a) and below the band in panel b) correspond to the local states formed predominantly of the defect orbital. 

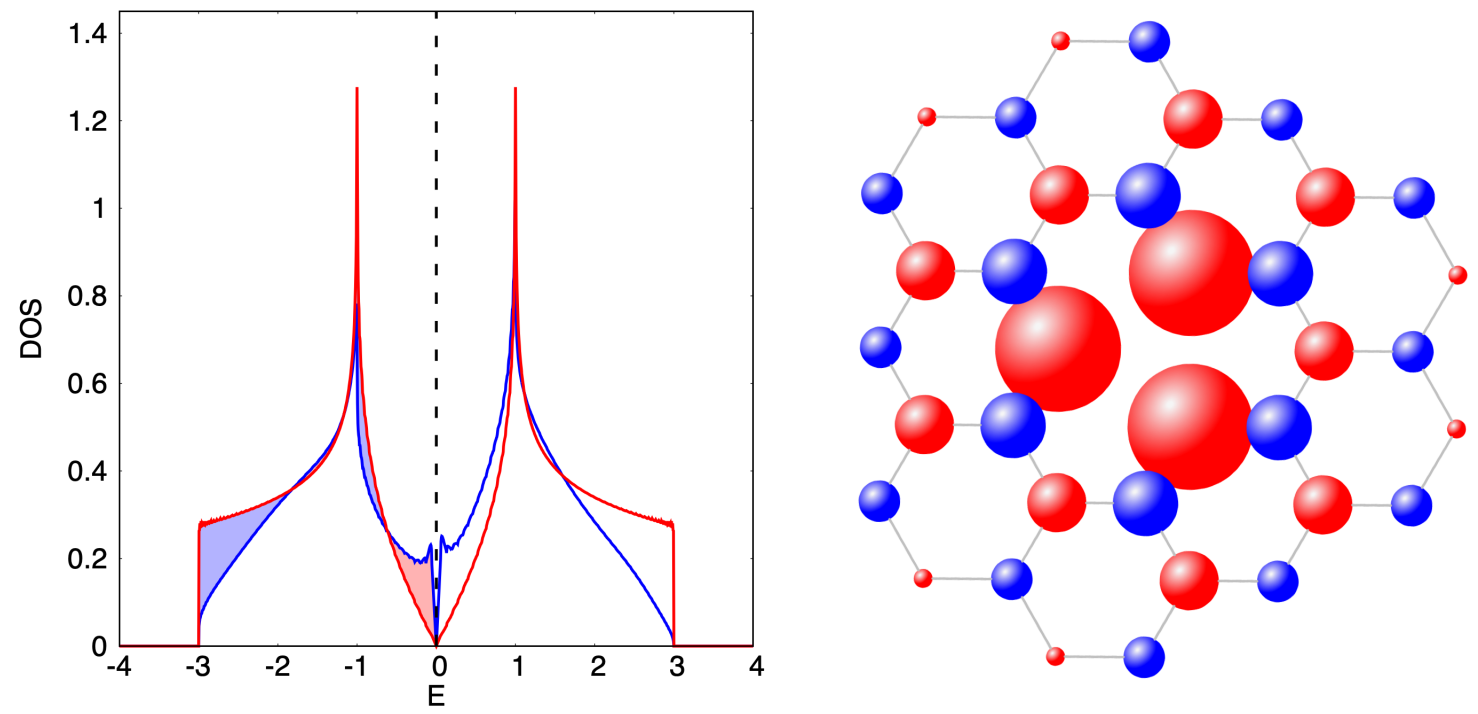

Figure 5: Perturbed diagonal DOS for the site nearest to the vacancy (left) and schematic illustration of the charge distribution caused by the vacancy defect in graphene. Legend for the plot and color scheme are the same as in previous Figures.

nearest neighbors. The perturbation itself reduces to nullifying the hopping matrix elements between the excluded site and its neighbors. The resulting charge distribution as well as AO-projected DOS for the site nearest to the vacancy are shown in Fig.5. Remarkably, the situation is quite different from that of the previous Subsection. For the predominantly off-diagonal perturbation, corrections to the diagonal densities are not that large $\left(Q_{1}=0.096, Q_{2}=-0.050, Q_{3}=0.045, Q_{4}=-0.032\right)$ and become negligible beyond $4^{\text {th }}$ neighbors from the vacancy. On the contrary, off-diagonal densities (bond orders) are affected much stronger: $\delta B_{01}=-0.525$ (quite obvious since the bond between the vacant site and its neighbor disappears), $\delta B_{12}=0.085, \delta B_{23}=-0.013, \delta B_{34}=0.012, \delta B_{45}=-0.020$ and all other changes occur only at a third decimal place. With a good accuracy (up to third decimal place) the following holds:

$$
\sum_{i \sim j} \delta B_{i j} \approx 3 \delta B_{01}+6 \delta B_{12}
$$

so that, all other oscillations of bond orders compensate each other and the total change of the bonding energy is a sum of two local terms - energy of three broken bonds and energies of six nearest bonds, which become stronger.

\section{Adsorption of hydrogen/alkali metal atom on graphene}

On-top adsorption of atomic hydrogen on graphene is a process of considerable interest in material science because it leads to a formation of $\mathrm{sp}^{3}$ defects, which usually present in synthetic graphene and affect its properties. It is known [73] that chemisorption of hydrogen forces carbon atom to rise above the plane by $0.4 \AA$ and to form a $\sigma$-bond with the adsorbate. This causes a change of hybridization state of the carbon atom (from $\mathrm{sp}^{2}$ to $\mathrm{sp}^{3}$ ) and consequent reorganization of both $\pi$ - and $\sigma$-systems - three $\pi$-bonds break and one new $\sigma$-bond appears along with distortion of three $\mathrm{C}-\mathrm{C} \sigma$-bonds. Complete treatment of such process can be performed in $\sigma-\pi$ approximation by considering $\pi$-bonds breaking as a vacancy forms (as described above) and a rigorous evaluation of the $\sigma$-system reorganization energy. Although theoretical basis for that has been already established in our previous works [56,74, 75, 76], full analysis of the problem goes beyond 
the scope of the present paper. Here, we restrict ourselves by testing the effect of interaction of "hydrogen" atom only with the graphene $\pi$-system, neglecting possible distortion of graphene geometry and not touching the $\sigma$-core. Treatment of the on-top adsorption on graphene in the framework of the standard Anderson impurity model was given in Refs. [78, 79, 80, 81, 82, 83]. Here we employ more advanced self-consistent model taking into account electron-electron interactions at different atomic sites.

Parameters required to describe interactions are calculated within the MNDO setting which has shown its validity for description of carbon allotropes [56]. We take $R(C-H)=1.1 \AA$. The adsorbate level lies below the Fermi level by $\varepsilon_{a}=-2.150$ and one-center electron-electron repulsion on the adsorbate $s$-orbital is $\gamma_{0}^{\prime}=3.952$. Two-center parameters for the $\mathrm{C}-\mathrm{H}$ pair have the following values $\beta^{\prime}=1.095$ and $\gamma_{1}^{\prime}=1.41$. All energies are in the units of $t$, which is known to be $2.4 \mathrm{eV}$ in graphene [77]. We denote the graphene site, interacting with the $\mathrm{H}$ atom, as " 0 ". The bare perturbation acts in the two-dimensional space $(0$-th $\pi$-AO of graphene and adsorbate $s$-orbital):

$$
\delta F=\left(\begin{array}{cc}
0 & -\beta^{\prime} \\
-\beta^{\prime} & 0
\end{array}\right)
$$

but the size of the cluster used for the self-consistent calculations is, of course, larger due to Coulomb interactions. To ensure we do not miss any significant changes in the density matrix elements, we, as previously, use the impurity cluster of $7 \times 7$ original graphene cells, whose unperturbed Greenian matrix was derived from the band structure calculated with the original unit cell of two atoms.

Perturbed AO-projected DOS for adsorbate:DOS $a(\varepsilon)$ and for the 0-th $\pi$-AO of graphene: $\operatorname{DOS}_{0}(\varepsilon)$ are plotted in Fig. 6. The adsorbate DOS below the Fermi level consists of two broad peaks. The first one lies in the interval $[-3,-1]$ with the maximum at $\varepsilon=\varepsilon_{a}$. It is obviously the adsorbate level broadened due to interaction with the graphene $\pi$-band. The second peak is much smaller than the first one and lies in the interval $[-1,0]$ with the maximum at $\varepsilon=-0.630$. At the point $\varepsilon=-1$ the DOS drops to zero ${ }^{8}$ due to the logarithmic singularity of the pure graphene DOS (GF). There is a local state above the band at $\varepsilon=3.090$, which does not contribute to the electronic density. No local states show up below the band in this case.

Adsorbate $s$-AO acquires a negative charge $Q_{a}=-0.7516$ and the site " 0 " a positive one of $Q_{0}=0.4607$. The charge distribution in graphene lattice generally follows the same pattern as in the impurity problem the values of the net-charges decay with the distance from the defect with alternating signs. The decay is rather slow and the charges become negligible $(<0.01)$ only beyond $6^{\text {th }}$ neighbors of the adsorption site. The net-charges of the neighbors from $1^{\text {st }}$ to $6^{\text {th }}$ are $Q_{1}=-0.3137, Q_{2}=0.1942, Q_{3}=-0.1355, Q_{4}=0.1174$, $Q_{5}=-0.0775$ and $Q_{6}=0.0538$. Due to the alternation of atomic charges, the contribution of two-center Coulomb interactions is negative, favoring the perturbed state.

Corrections to the bond orders decay much faster with the distance than atomic charges. Coulson bond order for the $\mathrm{C}-\mathrm{H}$ bond formed upon adsorption is $B_{a 0}=0.5802$. Three bonds of the adsorption site with its neighbors in the layer turn weaker $\left(\delta B_{01}=-0.1672\right)$, six bonds between $1^{\text {st }}$ and $2^{\text {nd }}$ neighbors turn stronger $\left(\delta B_{12}=0.0173\right)$ and twelve bonds between $2^{\text {nd }}$ and $3^{\text {rd }}$ neighbors again weaker $\left(\delta B_{23}=-0.01131\right)$. All further corrections to the bond orders are negligible $(<0.01)$ and do not contribute significantly to the adsorption energy. Due to the formation of the $\mathrm{C}-\mathrm{H}$ bond the system gains the energy:

$$
\delta E_{b}^{C-H}=-\left(2 \beta^{\prime}+\frac{\gamma_{1}^{\prime}}{2} B_{a 0}\right) B_{a 0}=-1.5080
$$

At the same time it loses the energy due to the weakening of three C-C bonds connected with the site "0":

$$
\delta E_{b}^{C-C}=-6 t \delta B_{01}=1.0032 .
$$

In total, the change in bonding energy is negative.

Electronic energy of chemisorption can be calculated by eq. (20) or alternatively one can adapt eq. (57), which in both cases gives the value of $\delta E=-1.392$ and favors the adsorption in agreement with DFT calculations [73]. However, numerical comparison of binding energies is not possible at the moment, since the calculated electronic energy does not include significant positive terms of core-core repulsion, which will be added in the next release. We, also, did not take into account rehybridization effects. The most significant negative contributions to the binding energy come from one-center term $(-1.62)$, two-center Coulomb and

\footnotetext{
${ }^{8}$ Since in practical calculations we do not have a genuine pole at $\varepsilon=-1$ and it is approximated by a peak with small yet finite width, the numerical DOS does not drop to zero exactly. However, this fact does not influence an integral of DOS.
} 


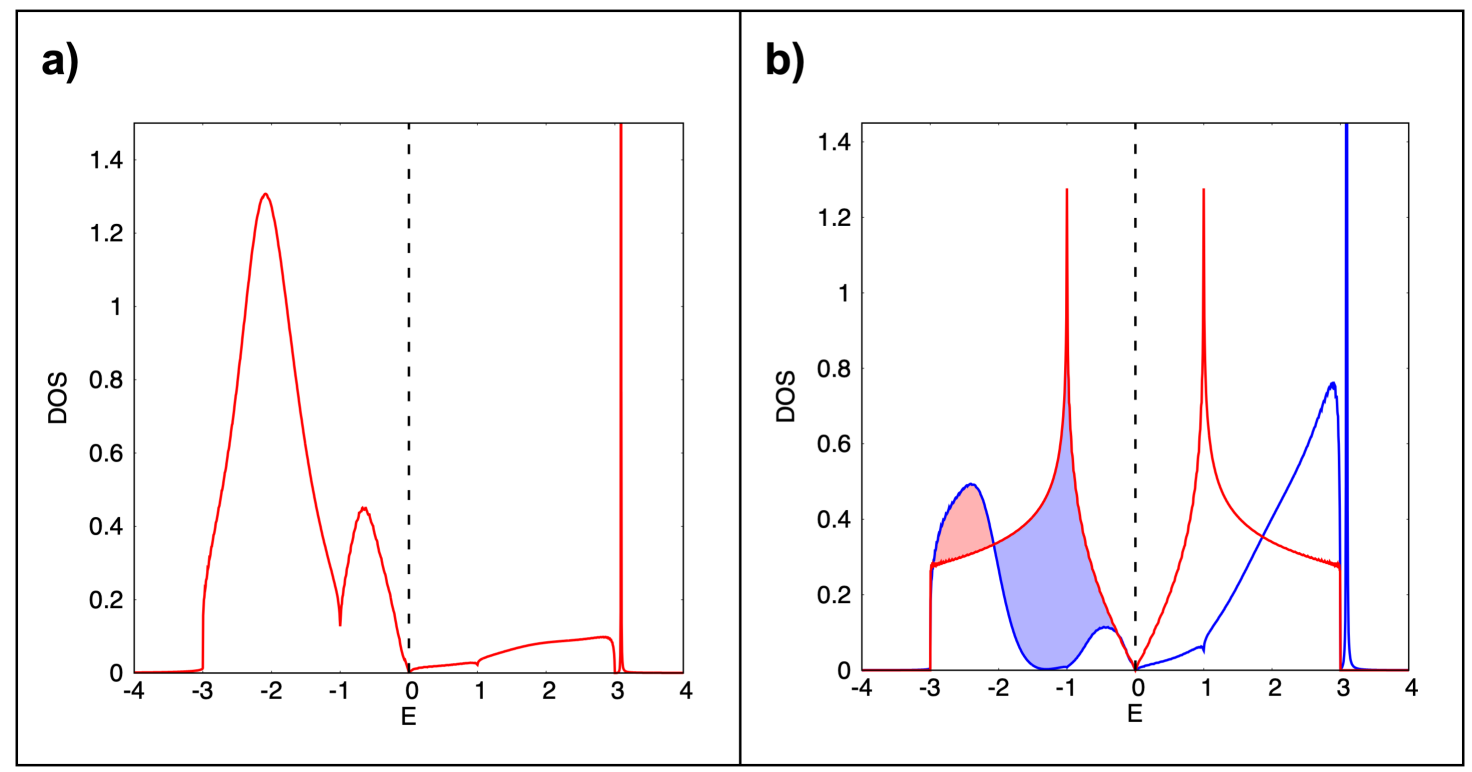

Figure 6: Diagonal AO-projected DOS for the atomic orbital of adsorbate (a) and for the orbital of graphene's site interacting with the $\mathrm{H}$ atom (b). On the right plot (b) red curve corresponds to the initial (unperturbed) DOS and the blue one - to the perturbed DOS. Narrow peaks on the plots above the band corresponds to the local state. On the plot a) one can also observe a virtual state being wide peak centered at the point ca. -0.5 .

bonding terms mentioned above. The most significant positive contribution comes from one-center repulsion of adsorbate electrons with different spin-projections which equals to $\gamma_{0}^{\prime} n_{a}^{2} / 4=1.7306$ (note, that we employ a non-magnetic solution for the perturbed system, but take into account that unperturbed hydrogen atom bears one unpaired electron).

In conclusion of this discussion, we mention that omitting the self-consistency procedure in the above model calculations leads to the charge distribution drastically differing from that obtained above. In the non self-consistent setting with the bare perturbation eq. (24), the following fast-decaying charge distribution $Q_{a}=-0.8372, Q_{0}=0.1103, Q_{1}=-0.0187, Q_{2}=0.0081$ is obtained (other charges are negligible). The local state below the band at $\varepsilon=-3.05$ appears, which is not there in the self-consistent calculations. This example demonstrates that in some cases the self-consistency is important, when considering point defects, not only quantitatively perspective, but also qualitatively.

\section{Conclusion}

We present the GoGreenGo package intended for description of point defects in crystals as well as for analysis of adsorption processes on surfaces. The package employs the Green's functions formalism in order to obtain the densities of states of the perturbed crystal containing defects. It builds the Green's function of the unperturbed crystal from the output produced by major $a b$ initio solid state quantum chemistry codes and solves the Dyson equation for the perturbed GF. Results of this calculation are processed so that the perturbed densities of states, charge distributions and the off-diagonal matrix elements of the density are available in the atomic basis. The package is tested for various local defects in model cubia crystals, in more realistic graphene and simple multiband model of $p$-cubium. In all cases, when the analytic solutions were available, the package manifested perfect agreement with the former. 


\section{Data Availability Statement}

The data that support the findings of this study are available either in Supplementary Materials or from the corresponding author upon reasonable request.

\section{Acknowledgments}

This work is supported by the State Task 0081-2019-0018 «Fundamental physicochemical laws of adsorption, adsorptive separation, adsorptive electrochemical ion exchange processes in nanoporous materials and fundamentals of targeted synthesis of new adsorbents».

\section{References}

[1] Tchougréeff, A.L. Phys. Chem. Chem. Phys. 1999, 1, 1051-1060.

[2] Pacchioni, G., Bagus, P.S., Parmigiani, F., Eds.; Cluster Models for Surface and Bulk Phenomena. NATO ASI Series (Series B: Physics), Springer: Boston, MA, 1992; Vol. 283, 682 pp.

[3] Tchougréeff, A.L. Hybrid Methods of Molecular Modeling. Springer Verlag, 2008; 346 pp.

[4] CRYSTAL software web-site. https://www.crystal.unito.it/ (accessed May 18, 2021).

[5] Blöchl, P.E. Phys. Rev. B. 1994, 50 (24), 17953-17978.

[6] Nørskov, J.K., Scheffler, M. and Toulhoat, H. MRS Bulletin. 2005, 31, 669-674.

[7] Toulhoat, H. Encyclopedia of Materials: Science and Technology (2nd Edition). 2010; p. 1-7.

[8] Hu, Q.-M., Reuter, K. and Scheffler, M. Phys. Rev. Lett. 2007, 98, 176103.

[9] Huang, C. and Carter, E.A. J. Chem. Phys. 2011, 135, 194104.

[10] Whitten, J.L. and Pakkanen, T.A. Phys. Rev. B. 1980, 21, 4357-4367.

[11] Izyumov, Yu.A. Adv. in Phys. 1965, 14, 569-619.

[12] Izyumov, Yu. A. and Medvedev, M. V. Soviet Physics JETP. 1965, 21, 381-388.

[13] Wolfram, T. and Calloway, J. Phys. Rev. 1963, 130, 2207.

[14] Anderson, P.W. Phys. Rev. 1961, 124, 41-53.

[15] Lifshitz, I.M. Soviet Physics JETP, 1947, 17, 1076.

[16] Newns, D.M. Phys. Rev. 1969, 178, 3.

[17] Muscat, J.P. and Newns, D.M. Prog. Surf. Sci. 1978, 9, 143.

[18] Newns, D.M. Phys. Rev. Lett. 1970, 25, 1575.

[19] Grimley, T.B. Proc. Phys. Soc. 1967, 90, 751.

[20] Grimley, T.B. J. Phys. C: Solid State Phys. 1970, 3, 1934.

[21] Grimley, T.B. Proc. Phys. Soc. 1967, 92, 776.

[22] Grimley, T.B. Progress in Surface and Membrane Science. 1975, 9, 71-161.

[23] Grimley, T.B. and Thorpe, B.J. Phys. Lett. 1971, 37A, 459.

[24] Grimley, T.B. and Pisani, C. J. Phys. C: Solid State Phys. 1974, 7, 2831. 
[25] Grimley, T.B. and Torrini, M. J. Phys. C: Solid State Phys. 1973, 6, 868.

[26] Einstein, T.L. and Schriefer, J. R. Phys. Rev. B. 1973, 7, 3629.

[27] Braun, O. M. and Medvedev, V.K. Usp. Fiz. Nauk. 1989, 157, 631-666.

[28] van Santen, R. A. Theoretical Heterogeneous Catalysis. In World Scientific Lecture and Course Notes in Chemistry. World Scientific, 1991; Vol. 5, 408 pp.

[29] McWeeny, R. and Sutcliffe, B.T. Methods of Molecular Quantum Mechanics (2-nd edition). Academic Press: London, 1992; 573 pp.

[30] Drabold, D.A., Estreicher, S.K. Defect Theory: An Armchair History. In Theory of Defects in Semiconductors; Drabold, D.A., Estreicher, S.K., Eds.; Springer-Verlag: Berlin, Heidelberg, 2007; pp 13-16.

[31] Zeller, R. DFT-based Green Function Approach for Impurity Calculations. In Correlated Electrons: From Models to Materials; Pavarini, E., Koch, E., Anders, F., M. Jarrell, M., Eds.; Forschungszentrum Jülich, 2012; 26 pp.

[32] GoGreenGo software web-site. https://netlab.cartesius.info/doxygen/GoGreenGo (accessed May 18, 2021).

[33] Zülicke, L. Quantenchemie. Grundlagen und allgemeine Methoden, Bd. 1. Deutscher Verlag der Wissenschaften: Berlin, 1973; 517 pp.

[34] GAUSSIAN software web-site. http://www.gaussian.com (accessed May 18, 2021).

[35] MOPAC software web-site. http://openmopac.net (accessed May 18, 2021).

[36] Mayer, I. Simple Theorems, Proofs, and Derivations in Quantum Chemistry. Kluwer Academic/Plenum Publishers: NY et al., 2003; 292 pp.

[37] Dyson, F.J. Phys. Rev. 1949, 75, 1736- 1755.

[38] Ashcroft, N. W., Mermin, N. D. Solid State Physics. Holt, Rinehart and Winston: New York, 1976; 826 pp.; Kittel, C. Introduction to Solid State Physics. Wiley: New York, 1996; Hoffmann, R. Solids and Surfaces: A Chemist's View of Bonding in Extended Structures. Wiley-VCH: New York, 1989; 152 pp.; Ziman, J. Principles of the Theory of Solids. Cambridge University Press, 1972; Dronskowski, R. Computational Chemistry of Solid State Materials. Wiley-VCH, Weinheim: New York, 2005; Bassani, F., Pastori Parravicini, G. Electronic States and Optical Transitions in Solids. Pergamon Press, 1975.

[39] Эварестов, Р.А. Квантовохимические методы в теории твердого тела. Изд-во Ленингр. ун-та: Ленинград, 1982; 280 pp. [in Russian]

[40] Deringer, V. L., Tchougreeff, A. L. and Dronskowski, R. J. Phys. Chem. A. 2011, 115, 5461; Maintz, S., Deringer, V. L., Tchougréeff, A. L. and Dronskowski, R. J. Comp. Chem. 2013, 34, 2557; Maintz, S., Deringer, V.L., Tchougréeff, A.L. and Dronskowski, R. J. Comp. Chem. 2016, 37, 1030 - 1035.

[41] Mostofi, A., Yates, J. R., Lee, Y.-S., Souza, I., Vanderbilt, D. and Marzari, D. Comput. Phys. Commun. 2008, 178, 685 .

[42] Aarons, J., Verga, L.G., Hine, N. D. M. and Skylaris, C.-K. Electron. Struct. 2019, 1, 035002.

[43] Hughbanks, T. and Hoffmann, R. J. Am. Chem. Soc. 1983, 105, 3528-3537; Hoffmann, R. Angew. Chem. Int. Ed. Engl. 1987, 26, 846-878; Steinberg, S. and Dronskowski, R. Crystals 2018, 8, 225; Dronskowski, R. and Blöchl, P.E. J. Phys. Chem. 1993, 97, 8617-8624.

[44] Tchougréeff, A.L. Lect. Notes Comp. Sci. 2019, 47, 639 - 651; see also https://cartesius.info/doxygen/index.html.

[45] HDF group web-site. https://portal.hdfgroup.org/ (accessed May 18, 2021). 
[46] Kresse, G. and Furthmüller, J. Phys. Rev. B 1996, 54, 11169-11186.

[47] ABINIT software web-site. https://www.abinit.org/ (accessed May 18, 2021).

[48] Plekhanov, E., Tchougréeff, A. and Dronskowski, R. Comp. Phys. Comm., 2020, 251, 107079; Plekhanov, E.A. and Tchougréeff, A.L. Comp. Mat. Sci., 2021, 188, 110140; Tchougréeff, A., Plekhanov, E. and Dronskowski, R. J. Comp. Chem., 2021, https://doi.org/10.1002/jcc.26561.

[49] Blöchl, P.E., Jepsen, O. and Andersen, O. K. Phys. Rev. B 1994, 49, 16223.

[50] Monkhorst, H. J. and Pack, J. D. Phys. Rev. B 1976, 13, 5188.

[51] Kronig, R. de L. J. Opt. Soc. Am. 1926, 12, 547-557.

[52] Bilato, R., Maj, O. and Brambilla, M. Adv. Comput. Math. 2014, 40, 1159-1168.

[53] Dewar, M. J. S. and Thiel, W. J. Am. Chem. Soc. 1977, 99, 4899-4907.

[54] Dewar, M. J. S., Zoebisch, E. G., Healy, E. F. and Stewart, J. J. P. J. Am. Chem. Soc. 1985, 107, 3902.

[55] Stewart, J. J. P. J. Comput. Chem. 1989, 10, 209-220.

[56] Popov, I.V., Görne, A.L., Tchougréeff, A.L. and Dronskowski, R. Phys. Chem. Chem. Phys. 2019, 21, $10961-10969$.

[57] Popov, I.V. and Tchougréeff, A.L. Theor. Chem. Acc. 2019, 138, 9.

[58] Bunge, C.F., Barrientos, J.A. and Bunge, A.V. At. Data Nucl. Data Tables 1993, 53, 113-162.

[59] Miguel, B., Koga, T. and García de la Vega, J. Theor. Chem. Acc. 2000, 104, 167-171.

[60] Burdett J.K. Prog. Solid St. Chem. 1984, 15, 173-255.

[61] Morita, T. and Horiguchi, T. J. Phys. Soc. Jap. 1971, 30, 957-964.

[62] Horiguchi, T. J. Phys. Soc. Jap. 1971, 30, 1261-1272.

[63] Morita, T. and Horiguchi, T. J. Math. Phys. 1971, 12.

[64] Lohrmann, D.J., Resca, L. Phys. Rev. B. 1989, 40, 8404-8409.

[65] Hadley, P., Tight binding. http://lampx.tugraz.at/ hadley/ss1/bands/tbtable/tbtable.html (accessed May 18, 2021).

[66] Губанов, В.А., Жуков, В.П., Литинский, А.О. Полуэмпирические методы молекулярных орбиталей в квантовой химии. Наука: Москва, 1976; 219 pp. [in Russian]

[67] Liu, H., Liu, Y. and Zhua, D. J. Mater. Chem. 2011, 21, 3335-3345.

[68] Lee, H., Paeng, K. and Kim, I.S. Synthetic Metals 2018, 244, 36-47.

[69] Castro Neto, A.H., Guinea, F., Peres, N. M. R., Novoselov, K.S. and Geim, A.K. Rev. Modern Phys. $\mathbf{2 0 0 9}, 81$.

[70] Gutman, I. Z. Naturforsch. 1981, 36a, 1112 - 1114; Coulson, C.A. and Longuet-Higgins, H. C. Proc. Roy. Soc. London 1947, A192, 16.

[71] Hückel, E. Z. Physikal. Chem. B 1937, 35, 163.

[72] Roberts, J. D., Caserio, M. C. Basic Principles of Organic Chemistry (2nd edition). W.A. Benjamin: Menlo Park, Calif., 1977.

[73] McKay, H., Wales, D.J., Jenkins, S.J., Verges, J.A. and de Andres, P.L. Phys. Rev. B. 2010, 81, 075425. 
[74] Tchougréeff, A.L. and Tokmachev, A.M. Int. J. Quant. Chem. 2006, 106, 571-587.

[75] Tokmachev, A.M. and Tchougréeff, A.L. J. Comp. Chem. 2001, 22, 752-764.

[76] Popov, I.V., Slavin, V.V., Tchougréeff, A.L. and Dronskowski, R. Phys. Chem. Chem. Phys. 2019, 21, $18138-18148$.

[77] Tchougréeff, A.L. and Hoffmann, R. J. Phys. Chem. 1992, 96, 8993.

[78] Davydov, S. Yu. Appl. Surf. Sci. 2010, 1506-1510.

[79] Davydov, S. Yu. Phys. Solid State 2011, 2545-2556.

[80] Davydov, S. Yu. Phys. Solid State 2012, 54, 1728-1731.

[81] Davydov, S. Yu. and Posrednik, O. V. Phys. Solid State 2015, 57, 1695-1698.

[82] Davydov, S. Yu., Sabirova, G. I. Tech. Phys. Lett. 2010, 36, 1154-1157.

[83] Davydov, S. Yu., Sabirova, G. I. Phys. Solid State 2011 53, 654-664.

[84] Шабат, Б. В. Введение в комплексный анализ. Наука: Москва, 1969; 577 pp. [in Russian]

[85] Gantmacher, F.R. The theory of matrices. Chelsea Pub. Co., 1960; Vol. 1, 374 pp.

\section{A Some elements of the general theory of GF}

Somewhat more refined explanation of relations between the GF and corresponding eigenvector-eigenvalue problem along with the separation of the GF into real and imaginary parts can be built upon the theory of residues of the functions of complex variable [84]. Since $\mathbf{G}(z)$ given by eqs. (4), (5) is continuous everywhere in the complex plane except for the simple, first-order poles located on the real axis at the points $z=\varepsilon_{\lambda}$, one can express the solution of the eigenvector-eigenvalue problem in terms of the GF. Its poles, obviously, correspond to the eigenvalues, while residues:

$$
\operatorname{Res}_{\varepsilon_{\lambda}} \mathbf{G}(z)=\frac{1}{2 \pi i} \oint_{C_{\varepsilon_{\lambda}}} \mathbf{G}(z) d z=\lim _{z \rightarrow \varepsilon_{\lambda}}\left(z-\varepsilon_{\lambda}\right) \mathbf{G}(z)=|\lambda\rangle\langle\lambda|
$$

are the projection operators to the eigenvectors of the Fockian F. In eq. (27) the first equality is the definition of the residue, the second one is the theorem expressing the integral over a contour encircling a simple pole, and the last one is the result of the calculation for the first-order poles. Correspondingly, the density (matrix) of the system in its ground state which is the sum of the operators projecting to the occupied $|\lambda\rangle$ 's expresses through the integral over the contour $C$ encircling all poles $\varepsilon_{\lambda} \leq \varepsilon_{F}$ Fig.7:

$$
\mathbf{P}=\sum_{\substack{\lambda \\ \varepsilon_{\lambda} \leq \varepsilon_{F}}}|\lambda\rangle\langle\lambda|=\frac{1}{2 \pi i} \oint_{C} \mathbf{G}(z) d z .
$$

The density matrix elements in the basis of atomic functions $|a\rangle,|b\rangle, \ldots$ can be found by integration of the matrix elements $G_{a b}$ of the GF over the contour $C$ encircling all poles $\varepsilon_{\lambda} \leq \varepsilon_{F}$ :

$$
P_{a b}=\frac{1}{2 \pi i} \oint_{C} G_{a b}(z) d z .
$$

In physics books (and in the main text) these results are frequently represented with use of the intuitively less clear recipe involving the $\delta$-"functions" and Kramers-Kronig relations as applied to the GF's of the real argument. It tacitly employs the crucial fact that in the expressions for the physical quantities the GF appears under the integral over the real axis, where it has a plenty of poles. Thus, the Green's functions 


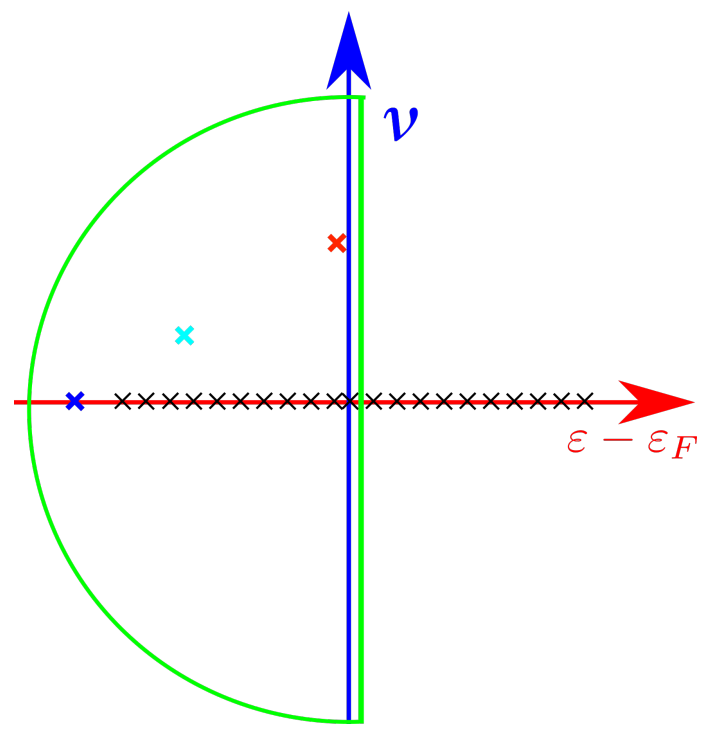

Figure 7: A form of the contour $C$ [29] used to calculate integrals of the GF - the elements of the perturbed density matrix. Black crosses are the poles of the unperturbed GF referring to the allowed energy band of the crystal. Blue cross on the abscissa to the left of the allowed band is the additional pole referring to the local state outside the allowed band which appeared due to perturbation - due to absence of intrinsic width it generates extremely narrow peaks in the DOS which can be seen in Figs. 4, 5. The cyan cross represents an additional pole within the allowed band. The corresponding virtual state provides a contribution to the perturbed DOS which has an energy spread proportional to the unperturbed DOS occurred at the energy corresponding real part of the pole. Similarly, the red cross represent a pole within the allowed energy band, but close to the Fermi level.

(on the real axis) are not functions in the usual sense anymore (although they are in the complex plane), rather they are distributions - linear functionals on the space of the true functions and the item of interest are the values of these functionals as such and the limits they may converge to. The reasoning is based on a high-school formula for the inverse of a complex number:

$$
z=x+i y \Rightarrow \frac{1}{z}=\frac{x-i y}{x^{2}+y^{2}},
$$

where $z$ is set equal to $\varepsilon+i \nu$ with an energy $\varepsilon$ and (half-)width $\nu$. Then GF separates into real and imaginary parts:

$$
\mathbf{G}(z)=\sum_{\lambda}|\lambda\rangle\langle\lambda|\left(\frac{\varepsilon-\varepsilon_{\lambda}}{\left(\varepsilon-\varepsilon_{\lambda}\right)^{2}+\nu^{2}}-i \frac{\nu}{\left(\varepsilon-\varepsilon_{\lambda}\right)^{2}+\nu^{2}}\right)=\Re \mathbf{G}(z)+i \Im \mathbf{G}(z) .
$$

The second term in the brackets above is nothing but the Lorentzian of half-width $\nu$ multiplied by $i \pi-$ one of the well-known probability (Cauchy) distributions. Making $\nu \rightarrow 0^{+}$nullifies it everywhere except for $\varepsilon=\varepsilon_{\lambda}$ where it diverges: very unnatural and peculiar behavior for a true function. Only, bearing in mind the intentional use of the above expression as of a multiplier in an integrand over $\varepsilon$ and not as a function of $\varepsilon$ per se, and then, taking the limit while $\nu \rightarrow 0^{+}$, supplies meaning to the formal expression:

$$
\lim _{\nu \rightarrow 0^{+}} \frac{1}{\varepsilon+i \nu-\varepsilon_{\lambda}}=\mathscr{P}\left(\frac{1}{\varepsilon-\varepsilon_{\lambda}}\right)-i \pi \delta\left(\varepsilon-\varepsilon_{\lambda}\right)
$$

abundant in physics books. It can be shown that if multiplied by a function $f(\varepsilon)$ then put under the integral and integrated first and making the $\nu \rightarrow 0^{+}$afterwards produces exactly the value of $f$ at $\varepsilon_{\lambda}: f\left(\varepsilon_{\lambda}\right)$ that what the Dirac $\delta$-function is expected to do. Analogously, treating the first term in the brackets as a multiplier to an integrand we arrive at the Hilbert transform of the function $f(\varepsilon)$. 


\section{B Matrix solution of the Dyson equation for local perturbation}

For the local perturbation $\mathbf{F}^{\prime}$ which affects only a subspace $P$ of the entire space of the one-electronic states it is natural to divide the latter into $P$ and its orthogonal complement $Q$. The matrix to be inverted in order to solve the Dyson equation then acquires a special form:

$$
\mathbf{I}-\mathbf{G}^{(0)} \mathbf{F}^{\prime}=\left(\begin{array}{cc}
\mathbf{I}_{P P}-\mathbf{G}_{P P}^{(0)} \mathbf{V} & 0 \\
-\mathbf{G}_{Q P}^{(0)} \mathbf{V} & \mathbf{I}_{Q Q}
\end{array}\right)
$$

where subscripts refer to the subspaces where the respective matrices act and the local perturbation $\mathbf{V}$ acts only in $P$ (is a $\operatorname{dim} P \times \operatorname{dim} P$ matrix). Although the matrix to be inverted has a very large (or even infinite) dimension of the entire orbital space of the crystal, its special structure permits to find its inverse relatively easily [85]:

$$
\left(\mathbf{I}-\mathbf{G}^{(0)} \mathbf{F}^{\prime}\right)^{-1}=\left(\begin{array}{cc}
\left(\mathbf{I}_{P P}-\mathbf{G}_{P P}^{(0)} \mathbf{V}\right)^{-1} & 0 \\
\mathbf{G}_{Q P}^{(0)} \mathbf{V}\left(\mathbf{I}_{P P}-\mathbf{G}_{P P}^{(0)} \mathbf{V}\right)^{-1} & \mathbf{I}_{Q Q}
\end{array}\right),
$$

that is, only a $\operatorname{dim} P \times \operatorname{dim} P$ matrix needs to be actually inverted (the rest is accessed through matrix multiplication). The correction to the unperturbed Greenian matrix caused by perturbation is then:

$$
\mathbf{G}-\mathbf{G}^{(0)}=\left(\begin{array}{cc}
\left(\mathbf{I}_{P P}-\mathbf{G}_{P P}^{(0)} \mathbf{V}\right)^{-1}-\mathbf{I}_{P P} & 0 \\
\mathbf{G}_{Q P}^{(0)} \mathbf{V}\left(\mathbf{I}_{P P}-\mathbf{G}_{P P}^{(0)} \mathbf{V}\right)^{-1} & 0
\end{array}\right)\left(\begin{array}{cc}
\mathbf{G}_{P P}^{(0)} & \mathbf{G}_{P Q}^{(0)} \\
\mathbf{G}_{Q P}^{(0)} & \mathbf{G}_{Q Q}^{(0)}
\end{array}\right)
$$

From the latter, one obtains equations given in the main text.

\section{Shift of the Fermi level}

The trace of the Greenian matrix after the perturbation changes as

$$
\delta \operatorname{Sp} \mathbf{G}(\varepsilon)=-\frac{\partial}{\partial \varepsilon} \ln \operatorname{det} \mathbf{G}(\varepsilon)+\frac{\partial}{\partial \varepsilon} \ln \operatorname{det} \mathbf{G}_{0}(\varepsilon)=\frac{\partial}{\partial \varepsilon} \ln \frac{\operatorname{det} \mathbf{G}_{0}(\varepsilon)}{\operatorname{det} \mathbf{G}(\varepsilon)} .
$$

The first equality comes from the fact, that determinant equals to the product of eigenvalues $g_{\lambda}(\varepsilon)$ of the Greenian matrix:

$$
\begin{gathered}
-\frac{\partial}{\partial \varepsilon} \ln \operatorname{det} \mathbf{G}(\varepsilon)=-\frac{\partial}{\partial \varepsilon} \ln \prod_{\lambda} g_{\lambda}(\varepsilon)=-\lim _{\nu \rightarrow 0^{+}} \sum_{\lambda} \frac{\partial}{\partial \varepsilon} \ln \left(\varepsilon+i \nu-\varepsilon_{\lambda}\right)^{-1}= \\
=\lim _{\nu \rightarrow 0^{+}} \sum_{\lambda}\left(\varepsilon+i \nu-\varepsilon_{\lambda}\right)^{-1}=\sum_{\lambda} g_{\lambda}(\varepsilon)=\operatorname{SpG}(\varepsilon)
\end{gathered}
$$

From the Dyson's equation we get:

$$
\operatorname{det} \mathbf{G}(\varepsilon)=\operatorname{det}\left(\mathbf{I}-\mathbf{G}^{(0)}(\varepsilon) \mathbf{F}^{\prime}\right)^{-1} \operatorname{det} \mathbf{G}^{(0)}(\varepsilon) .
$$

The latter allows to express the trace correction as

$$
\delta \operatorname{Sp} \mathbf{G}(\varepsilon)=\frac{\partial}{\partial \varepsilon} \ln \operatorname{det}\left(\mathbf{I}-\mathbf{G}^{(0)}(\varepsilon) \mathbf{F}^{\prime}\right)
$$

If the Fermi level had had not changed, the conservation of number of electrons would require that

$$
-\frac{1}{\pi} \int_{-\infty}^{\varepsilon_{F}} \frac{\partial}{\partial \varepsilon} \Im \ln \operatorname{det}\left(\mathbf{I}-\mathbf{G}^{(0)}(\varepsilon) \mathbf{F}^{\prime}\right) d \varepsilon=0
$$


which is, generally, not satisfied. Let us denote the integral on the left of the last equation as $\Delta n$. The condition of conservation of number of electrons reads then

$$
\mathrm{K} \int_{\varepsilon_{F}}^{\varepsilon_{F}+\delta \varepsilon_{F}} \operatorname{DOS}_{0}(\varepsilon) d \varepsilon=-\Delta n
$$

where $\operatorname{DOS}_{0}(\varepsilon)$ is a sum of diagonal projected DOS for a single unit cell in the initial (unperturbed) system. From this equation one can see that for $\mathrm{K} \rightarrow \infty$ the shift of the Fermi level approaches zero. However, in the finite dimension models this shift needs to be taken into account especially in the case of small $\mathrm{K}$ (not very dense $k$-mesh). Note, that for negative $\Delta n$ the Fermi level shifts up, while for positive - down.

\section{Pole structure of the perturbed GF}

Due to a specific role which poles of the GF play throughout the integration prescribed by eq. (29), the qualitative effect of perturbation crucially depends on whether it causes any additional poles to appear on the right hand side of eq. (14). A sketch of evolving events comes from considering simplest perturbation $v$, acting on the one-dimensional subspace $P$ spanned by the state $|0\rangle$. The $1 \times 1$ matrix to be inverted to solve the Dyson equation (see Appendix B) is

$$
1-v G_{00}^{(0)}
$$

and the only nonvanishing matrix element of the mass operator eq. (13) takes the form:

$$
M_{00}=\frac{v}{1-v G_{00}^{(0)}} .
$$

The tentative additional poles are zeroes of the denominator in the latter equation. Separating $G_{00}^{(0)}$ into real and imaginary parts we obtain an equation for the poles:

$$
1-v \Re G_{00}^{(0)}\left(\varepsilon_{l}\right)-i v \Im G_{00}^{(0)}\left(\varepsilon_{l}\right)=0
$$

For the complex quantity to vanish its real and imaginary parts have to vanish separately. The imaginary part $\Im G_{00}^{(0)}(\varepsilon)$ is zero everywhere in the forbidden energy bands of a crystal (where the DOS vanishes). In this case only one condition needs to be satisfied:

$$
\Re G_{00}^{(0)}\left(\varepsilon_{l}\right)=\frac{1}{v}
$$

at a point $\varepsilon_{l}$ outside the allowed energy band. Thisdefines position of the local state and is referred to in the main text throughout the discussion of additional poles in the case of substitutional defect in cubia. Here we only mention that for cubia $\Re G_{00}^{(0)}$ is positive above the Fermi level and negative below it (see Supporting Materials Sec.1), therefore a positive perturbation $(v>0)$ can only lead to a local state above the allowed band, while a negative one - only below it. Values of $v$ required to satisfy the condition for cubia are discussed in the main text.

If eq. (43) is satisfied for the complex point $\varepsilon_{v}+i \nu_{v}$ with its real part $\varepsilon_{v}$ lying inside the band $\left(\Im G_{00}^{(0)}\left(\varepsilon_{v}\right) \neq\right.$ 0 ), then a virtual state appears - a pole in the complex plane. It manifests itself on the real axis as a maximum of the DOS in the allowed energy band [11]. Behavior of $\operatorname{DOS}_{0}(\varepsilon)$ in the vicinity of the virtual state $\varepsilon_{v}$ derives from the following equation for the perturbed GF with mass operator eq. (41):

$$
G_{00}(\varepsilon)=G_{00}^{(0)}(\varepsilon)+G_{00}^{(0)}(\varepsilon) M G_{00}^{(0)}(\varepsilon)=\frac{G_{00}^{(0)}(\varepsilon)}{1-v G_{00}^{(0)}(\varepsilon)} .
$$

Evaluating imaginary part of this, one obtains

$$
\operatorname{DOS}_{0}(\varepsilon)=-\pi^{-1} \frac{\Im G_{00}^{(0)}(\varepsilon)}{\left(1-v \Re G_{00}^{(0)}(\varepsilon)\right)^{2}+v^{2}\left(\Im G_{00}^{(0)}(\varepsilon)\right)^{2}} .
$$


If $\Im G_{00}^{(0)}(\varepsilon)$ changes slowly in the vicinity of $\varepsilon_{v}$, the perturbed density of states is proportional to a Lorentzian:

$$
\operatorname{DOS}_{0}(\varepsilon) \sim \frac{\pi^{-1} \eta}{\left(\varepsilon-\varepsilon_{v}\right)^{2}+\eta^{2}}
$$

with a half-width

$$
\eta=\frac{\Im G_{00}^{(0)}\left(\varepsilon_{v}\right)}{\partial \Re G_{00}^{(0)}(\varepsilon) /\left.\partial \varepsilon\right|_{\varepsilon=\varepsilon_{v}}}
$$

which follows from the expansion

$$
1-v \Re G_{00}^{(0)}(\varepsilon)=-\left.v\left(\varepsilon-\varepsilon_{v}\right) \frac{\partial \Re G_{00}^{(0)}(\varepsilon)}{\partial \varepsilon}\right|_{\varepsilon=\varepsilon_{v}} .
$$

If the half-width $\nu \ll \varepsilon_{v}$, one would observe a sharp maximum of DOS at $\varepsilon_{v}$, otherwise, there would not be a definite peak. In addition, from this analysis one can explicitly observe that for the pole outside the band the half-width vanishes $(\eta=0)$ yielding a discrete peak in DOS, which corresponds to the local state discussed above.

It should be noted that the condition eq. (42) may not be possible to satisfy for a given value of perturbation $v$. In this case there are no local or virtual states influencing the resulting DOS, and its form is simply given by eq. (44).

In the general case a similar analysis of the pole structure can be performed if the mass operator $\mathbf{M}$ is expressed according to Kramer's rule through the adjugate matrix and determinant $D=\operatorname{det}\left(\mathbf{I}_{P P}-\mathbf{G}_{P P}^{(0)} \mathbf{V}\right)$ :

$$
\mathbf{M}=\frac{\operatorname{Vadj}\left(\mathbf{I}_{P P}-\mathbf{G}_{P P}^{(0)} \mathbf{V}\right)}{D} .
$$

The sought additional poles are the roots of $D$, which is a polynomial of the power $\operatorname{dim} P$. If a $\operatorname{root}$ of determinant $z_{0}=\varepsilon_{l}$ is real, then corresponding pole describes a local state. If it is complex then it refers to a virtual state. In the impurity problems local states appear outside the band where $\Im D=0$ for all points and position of the local state is therefore determined by roots of $\Re D$. Positions of the virtual states are determined by zeroes of $\Re D$ as well, but located inside the band [11].

While virtual states do not provide any difficulties in evaluating integrals eqs. (19) and (16), the local states do. In their presence those integrals are understood in terms of Cauchy principal value. Contribution of the local state outside the band to the density matrix elements can be evaluated by considering residue at $z_{0}$, which for the root of the first order has a form

$$
\operatorname{Res}_{z_{0}} G_{a b}(z)=\lim _{z \rightarrow z_{0}}\left(z-z_{0}\right) G_{a b}(z)=\langle a \mid l\rangle\langle l \mid b\rangle
$$

From eq. (48) it follows that the perturbed function $G_{a b}$ may be represented in a form $f(z) / D(z)$. If both $D$ and $f$ are holomorphic and $D^{\prime}\left(z_{0}\right) \neq 0$ then the residue can be evaluated as

$$
\operatorname{Res}_{z_{0}}^{\sigma} G_{a b}(z)=\frac{f\left(z_{0}\right)}{D^{\prime}\left(z_{0}\right)}
$$

This allows to express contributions of localized states to the density matrix elements through the derivatives of $D$. With the same approach it may be shown that local state outside the band contributes to the energy correction $\delta \varepsilon$ as $\varepsilon_{l}$.

\section{E Model periodic systems}

In order to test our approach we consider impurity atoms in several simple model metals: simple cubium, face centered cubium and body centered cubium each bearing one s-orbital per lattice node. Besides that 
we consider perturbations of $\pi$-bands of graphene. All these lattices have one electron per site. We consider them in the framework of HF approach with extended Hubbard Hamiltonian:

$$
\begin{aligned}
H=- & U \sum_{i, \sigma} c_{i \sigma}^{+} c_{i \sigma}-m \gamma_{1} \sum_{i, \sigma} c_{i \sigma}^{+} c_{i \sigma}-\beta \sum_{i \sim j, \sigma}\left\{c_{i \sigma}^{+} c_{j \sigma}+\text { h.c. }\right\}+ \\
& +\gamma_{0} \sum_{i} c_{i \alpha}^{+} c_{i \alpha} c_{i \beta}^{+} c_{i \beta}+\gamma_{1} \sum_{i \sim j, \sigma, \kappa} c_{i \sigma}^{+} c_{i \sigma} c_{j \kappa}^{+} c_{j \kappa}
\end{aligned}
$$

featuring following energy contributions: one-center core attraction (term proportional to $U$ ), one-center Coulomb repulsion $\left(\gamma_{0}\right)$, nearest neighbor electron hopping $(\beta)$ and Coulomb repulsion $\left(\gamma_{1}\right)$; the term proportional to $m$ the number of neighbors of each atom in respective lattice models the core attraction to the nearest neighbors which is necessary for overall energy balance. For spin and charge symmetric states we set

$$
\begin{gathered}
\left\langle c_{i \alpha}^{+} c_{i \alpha}\right\rangle=\left\langle c_{i \beta}^{+} c_{i \beta}\right\rangle=\frac{1}{2}, \\
\left\langle c_{i \alpha}^{+} c_{j \alpha}\right\rangle=\left\langle c_{i \beta}^{+} c_{j \beta}\right\rangle=\frac{B}{2},
\end{gathered}
$$

where the last equation holds for neighbor sites $i$ and $j$. In the Hartree-Fock approximation the following Fockian appears:

$$
F=\alpha \sum_{i} c_{i \sigma}^{+} c_{i \sigma}-t \sum_{i \sim j}\left\{c_{i \sigma}^{+} c_{j \sigma}+h . c .\right\}
$$

where $\alpha$ and $t$ are:

$$
\begin{gathered}
\alpha=-U-m \gamma_{1}+\gamma_{0}\left\langle c_{i \alpha}^{+} c_{i \alpha}\right\rangle+\gamma_{1} \sum_{j \sim i} \sum_{\sigma}\left\langle c_{j \sigma}^{+} c_{j \sigma}\right\rangle=-U+\frac{\gamma_{0}}{2}, \\
t=\beta+\gamma \frac{B}{2} .
\end{gathered}
$$

In all further discussions we set the energy reference at $\alpha=0$. In principle these equations are to be solved iteratively for the only nontrivial density matrix element $B$. However, in cubia and graphene the bond order $B$ does not depend on Hamiltonian parameters due to the symmetry and no self-consistent procedure is required to obtain analytical solutions in these cases.

In some cases to get anlytical estimates we will be interested in a simpler model without two-center Coulomb terms in Hamiltonian (simple Hubbard Hamiltonian). Then $t$ does not depend on the density matrix elements.

The energy of the defect formation can be expressed through the parameters of the Hamiltonian and the density matrix elements corrections as

$$
\begin{aligned}
\delta E=\{v+ & \left.\frac{\delta \gamma_{0}}{4} \delta n_{0}\right\} n_{0}-2 \delta t \sum_{i \sim 0} B_{i 0}+\delta \gamma_{1} \sum_{i \sim 0}\left(\delta n_{0} \delta n_{i}-1\right)-2 t \sum_{i \sim j} \delta B_{i j}+ \\
& +\frac{\gamma_{1}}{2} B \sum_{i \sim j} \delta B_{i j}+\frac{\gamma_{0}}{4} \sum_{i} \delta n_{i}^{2}+\gamma_{1} \sum_{i \sim j} \delta n_{i} \delta n_{j}-\Delta n \varepsilon_{F} .
\end{aligned}
$$

This equation has an advantage that it allows to analyze contributions of different terms and it is being referred to in the main text in the discussions of the defects' energies. 